DEPT. OF MATH. UNIV. OF OSLO

ISSN 0806-2439 JANUARY 2005

\title{
TRANSFER MAPS AND THE CYCLOTOMIC TRACE
}

\author{
CHRISTIAN SCHLICHTKRULL
}

\begin{abstract}
We analyze the equivariant restriction (or transfer) maps in topological Hochschild homology associated to inclusions of group rings of the form $R[H] \rightarrow R[G]$, where $R$ is a symmetric ring spectrum, $G$ is a discrete group and $H \subseteq G$ is a subgroup of finite index. This leads to a complete description of the associated restriction (or transfer) maps in topological cyclic homology
\end{abstract}

$$
\operatorname{Res}_{G}^{H}: \operatorname{TC}(R[G]) \rightarrow \mathrm{TC}(R[H])
$$

in terms of the well-known stable transfers in equivariant stable homotopy theory. More generally, we analyze the restriction maps encountered in connection with monoid rings such as polynomial rings and truncated polynomial rings. As a first application of these results we prove a conjecture by Bökstedt, Hsiang and Madsen on how the transfer maps in Waldhausen's algebraic K-theory of spaces relate to the transfers in the stable equivariant homotopy category of a finite cyclic group. As a second application we calculate the subgroup of transfer invariant homotopy classes

$$
\pi_{*} \mathrm{TC}\left(R\left[z_{1}, z_{1}^{-1}, \ldots, z_{m}, z_{m}^{-1}\right]\right)^{\mathrm{INV}}
$$

and we show that the TC-analogue of the lower K-groups vanish below degree -1 .

\section{INTRODUCTION}

Let $R$ be a connective symmetric ring spectrum. Given a discrete group $G$, the associated symmetric group ring spectrum is defined by $R[G]=R \wedge G_{+}$. When $R$ is the Eilenberg-Mac Lane spectrum $H \bar{R}$ associated to a discrete ring $\bar{R}$, this is equivalent to the Eilenberg-Mac Lane spectrum $H \bar{R}[G]$. In general, $\pi_{*} R[G]$ is the ordinary group ring of $G$ with coefficients in $\pi_{*} R$. In this paper we analyze the restriction (or transfer) maps in topological cyclic homology

$$
\operatorname{Res}_{G}^{H}: \operatorname{TC}(R[G]) \rightarrow \mathrm{TC}(R[H])
$$

associated to inclusions of the form $R[H] \rightarrow R[G]$, where $H \subseteq G$ is a subgroup of finite index. These restriction maps are related via the cyclotomic trace to the analogous restriction (or transfer) maps in algebraic K-theory

Date: January 14, 2005. 
by a commutative diagram

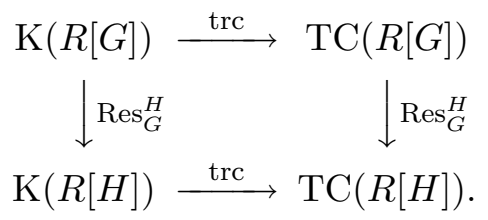

We recall the definition of the cyclotomic trace trc and prove the commutative of the above diagram in Appendix A. In the case where $R$ is an Eilenberg-Mac Lane spectrum we recover the transfer map in Quillen's algebraic K-theory on the left hand side. When $R$ is the sphere spectrum, we recover the transfer map in Waldhausen's algebraic K-theory of spaces $A(B G) \rightarrow A(B H)$, see [31].

The cyclotomic trace was introduced in $[8]$ and has proved to be a strong invariant of algebraic K-theory, [14], [21], [9], leading to many explicit calculations. By the above diagram it follows that it also provides a strong tool for analyzing the transfer maps in algebraic K-theory. More generally, our analysis covers the transfer maps encountered in connection with monoid rings such as polynomial rings and truncated polynomial rings. We include two applications of the general theory. The first is to prove a conjecture by Bökstedt, Hsiang and Madsen [8, 7.14] on how the transfers in Waldhausen's algebraic K-theory of spaces relate to the stable transfers in the $C_{r}$-equivariant stable homotopy category for a finite cyclic group $C_{r}$. The second application is a calculation of the TC-transfer invariant homotopy classes of Laurent polynomial rings. Other applications of the results of this paper appear in $[21,5.1]$ where they are used in the calculation of $\mathrm{K}_{*}\left(\mathbb{F}_{p}\left[C_{p^{n}}\right]\right)$ and in [5] where they are used in the analysis of Bloch's curves on algebraic K-theory. The restriction map in topological Hochschild homology was also considered in [26], but the analysis presented here goes much further.

We first give an overview of the general methods and results of this paper. The framework in [8] is not quite structured enough for the analysis given here and we shall present an account of what we find is a convenient choice of details. This also lays the foundation for further work in this area such as the analysis of the curves on the algebraic K-theory of a general ring spectrum.

1.1. Topological cyclic homology and restriction maps. Given a symmetric ring spectrum $R$, we let $\mathrm{TH}(R)$ be the topological Hochschild homology spectrum of the skeleton category $\mathcal{F}(R)$ of finitely generated free $R$-modules. By definition, this is the topological realization of a cyclic spectrum providing it with a canonical action of the circle group $\mathbb{T}$. It further has the structure of a cyclotomic spectrum, which implies the existence of two families of spectrum maps

$$
F_{s}, R_{s}: \operatorname{TH}(R)^{C_{r s}} \rightarrow \mathrm{TH}(R)^{C_{r}},
$$


relating the fixed-point spectra for the finite cyclic groups $C_{r s}$ and $C_{r}$. This makes the fixed-point spectra $r \mapsto \mathrm{TH}(R)^{C_{r}}$ a diagram over a certain category $\mathbb{I}$ and by definition

$$
\mathrm{TC}(R)=\underset{\mathbb{I}}{\operatorname{holim}} \mathrm{TH}(T)^{C_{r}} .
$$

Suppose now that $H \subseteq G$ is a subgroup of finite index and consider the restriction functor

$$
\operatorname{Res}_{G}^{H}: \mathcal{F}(R[G]) \rightarrow \mathcal{F}(R[H]) .
$$

Since we work with skeleton categories this requires the choice of a transversal of $H$ in $G$. The functors associated to two such choices are related by a natural isomorphism. Since the TH-construction is functorial with respect to such functors this induces a restriction (or transfer) map

$$
\operatorname{Res}_{G}^{H}: \operatorname{TH}(R[G]) \rightarrow \mathrm{TH}(R[H]) .
$$

This is a map of cyclotomic spectra and the TC restriction map is obtained by passing to the homotopy limit over $\mathbb{I}$ of the induced maps of fixed-point spectra. In order to analyze it we must therefore identify the induced maps of fixed-point spectra in a way that is compatible with the cyclotomic structure. On the level of homotopy groups we prove that the action of $\operatorname{Res}_{G}^{H}$ can be described in terms of the homology transfers for the equivariant homology theory $\mathrm{H}_{*}^{C_{r}}(-, \mathrm{TH}(R))$ whose definition we recall in Section 6.2. Let $\langle G\rangle$ and $\langle H\rangle$ denote the sets of conjugacy classes of elements in $G$ and $H$, respectively. There are isomorphisms

$$
\pi_{*} \mathrm{TH}(R[G])^{C_{r}} \simeq \bigoplus_{\omega \in\langle G\rangle} \mathrm{H}_{*}^{C_{r}}\left(B C_{G}(\omega) ; \mathrm{TH}(R)\right)
$$

and

$$
\pi_{*} \mathrm{TH}(R[H])^{C_{r}} \simeq \bigoplus_{\lambda \in\langle H\rangle} \mathrm{H}_{*}^{C_{r}}\left(B C_{H}(\lambda) ; \mathrm{TH}(R)\right)
$$

where $C_{G}(\omega)$ and $C_{H}(\lambda)$ denote the centralizers associated to choices of representatives in $\omega$ and $\lambda$. The homology groups associated to different choices are canonically isomorphic.

Theorem 1.1. Using the above isomorphisms, the action of $\operatorname{Res}_{G}^{H}$ on homotopy groups is described by the $\langle H\rangle \times\langle G\rangle$-matrix $\{\operatorname{Trf}(\lambda, \omega)\}$, where

- if $\lambda \nsubseteq \omega$, then $\operatorname{Trf}(\lambda, \omega)=0$;

- if $\lambda \subseteq \omega$ and $x \in \lambda$ is a representative, then

$$
\operatorname{Trf}(\lambda, \omega): \mathrm{H}_{*}^{C_{r}}\left(B C_{G}(x), \mathrm{TH}(R)\right) \rightarrow \mathrm{H}_{*}^{C_{r}}\left(B C_{H}(x), \mathrm{TH}(R)\right)
$$

is the homology transfer associated to the "covering up to homotopy" $B C_{H}(x) \rightarrow B C_{G}(x)$.

This theorem follows immediately by combining the more precise spectrum level results in Theorem 1.2 and Theorem 1.4, which we discuss next. By definition, a map of cyclotomic spectra is an equivalence if it induces an equivalence on $C_{r}$ fixed-point spectra for all $r$. Such an equivalence induces 
an equivalence of the associated homotopy limits over $\mathbb{I}$. We usually prefer to work equivariantly, keeping track of the cyclotomic structures and passing to fixed point spectra only at the last stage.

1.2. Separating $R$ and $G$. The first step is to separate the ring spectrum $R$ from the groups $G$ and $H$. This requires that we view $\mathrm{TH}(R)$ as a $\mathbb{T}$ spectrum indexed on a complete set $\mathcal{V}$ of finite dimensional representations. Let $\mathbb{S}$ be the sphere spectrum. The pairing $\mathbb{S}[G] \wedge R \rightarrow R[G]$ of symmetric ring spectra induces an equivalence of cyclotomic spectra

$$
\mathrm{TH}(\mathbb{S}[G]) \wedge \mathcal{V} \mathrm{TH}(R) \stackrel{\sim}{\rightarrow} \mathrm{TH}(R[G])
$$

where $\wedge \mathcal{V}$ denotes the smash product of $\mathbb{T}$-spectra indexed on $\mathcal{V}$, see Section 2.3. This equivalence is natural with respect to restriction maps, hence it suffices to analyze the latter for inclusions of spherical group rings of the form $\mathbb{S}[H] \rightarrow \mathbb{S}[G]$. In order to do this we introduce the combinatorial restriction map

$$
\operatorname{Res}_{G}^{H}: \mathbb{E} \Sigma_{\mathbb{T}}^{\infty}\left(B^{\mathrm{cy}}(G)_{+}\right) \rightarrow \mathbb{E} \Sigma_{\mathbb{T}}^{\infty}\left(B^{\mathrm{cy}}(H)_{+}\right),
$$

where $B^{\text {cy }}$ is Waldhausen's cyclic bar construction and $\mathbb{E} \Sigma_{\mathbb{T}}^{\infty}$ is a cyclotomic version of the combinatorial Barratt-Eccles model of the suspension spectrum. This is a map of cyclotomic spectra and provides a model for the TH-restriction map in the sense that there is a chain of equivalences of cyclotomic spectra

$$
\mathrm{TH}(\mathbb{S}[G]) \simeq \mathbb{E} \Sigma_{\mathbb{T}}^{\infty}\left(B^{\mathrm{cy}}(G)_{+}\right)
$$

in which each equivalence is natural with respect to restriction maps. The cyclic bar construction splits as a disjoint sum of components $B^{\text {cy }}(G, \omega)$ indexed on the set of conjugacy classes $\langle G\rangle$ and there results an equivalence of cyclotomic spectra

$$
\mathbb{E} \Sigma_{\mathbb{T}}^{\infty}\left(B^{\mathrm{cy}}(G)_{+}\right) \stackrel{\sim}{\rightarrow} \prod_{\omega \in\langle G\rangle}^{\prime} \mathbb{E} \Sigma_{\mathbb{T}}^{\infty}\left(B^{\mathrm{cy}}(G, \omega)_{+}\right),
$$

where $\prod^{\prime}$ denotes the weak product, that is, the colimit of the finite products. Let $H \subseteq G$ be a subgroup of finite index as above. There is a similar splitting of $B^{\text {cy }}(H)$ and for each pair of conjugacy classes $\lambda \in\langle H\rangle$ and $\omega \in\langle G\rangle$, we let

$$
\operatorname{Res}_{G}^{H}(\lambda, \omega): \mathbb{E} \Sigma_{\mathbb{T}}^{\infty}\left(B^{c y}(G, \omega)_{+}\right) \rightarrow \mathbb{E} \Sigma_{\mathbb{T}}^{\infty}\left(B^{c y}(H, \lambda)_{+}\right)
$$

be the map of $\mathbb{T}$ spectra obtained by composing $\operatorname{Res}_{G}^{H}$ with the inclusion and projection specified by $\lambda$ and $\omega$. Combining the equivalences described above, the behavior of the restriction maps under these splittings is summarized in the following theorem. 
Theorem 1.2. A subgroup $H \subseteq G$ of finite index gives rise to a diagram of cyclotomic spectra

$$
\begin{aligned}
& \mathrm{TH}(R[G]) \stackrel{\sim}{\longrightarrow} \prod_{\omega \in\langle G\rangle}^{\prime} \mathbb{E} \Sigma_{\mathbb{T}}^{\infty}\left(B^{c y}(G, \omega)_{+}\right) \wedge_{\mathcal{V}} \mathrm{TH}(R) \\
& \downarrow \operatorname{Res}_{G}^{H} \quad \downarrow \prod_{\omega \in\langle G\rangle}^{\prime} \prod_{\lambda \subseteq \omega}^{\prime} \operatorname{Res}_{G}^{H}(\lambda, \omega) \wedge \mathrm{id} \\
& \mathrm{TH}(R[H]) \stackrel{\sim}{\longrightarrow} \prod_{\lambda \in\langle H\rangle}^{\prime} \mathbb{E} \Sigma_{\mathbb{T}}^{\infty}\left(B^{c y}(H, \lambda)_{+}\right) \wedge \mathcal{V} \mathrm{TH}(R),
\end{aligned}
$$

in which the horizontal arrows represent chains of equivalences of cyclotomic spectra each of which commute strictly with the restriction maps.

The vertical map on the right hand side is a product over $\omega \in\langle G\rangle$ of maps of the form

$$
\mathbb{E} \Sigma_{\mathbb{T}}^{\infty}\left(B^{\mathrm{cy}}(G, \omega)_{+}\right) \wedge_{\mathcal{V}} \mathrm{TH}(R) \rightarrow \prod_{\lambda \subseteq \omega}^{\prime} \mathbb{E} \Sigma_{\mathbb{T}}^{\infty}\left(B^{\mathrm{cy}}(H, \lambda)_{+}\right) \wedge_{\mathcal{V}} \mathrm{TH}(R),
$$

where $\lambda$ runs over the $H$-conjugacy classes contained in $\omega$ and the $\lambda$ th component is given by $\operatorname{Res}_{G}^{H}(\lambda, \omega) \wedge$ id. In particular, $\operatorname{Res}_{G}^{H}(\lambda, \omega)$ is trivial when $\lambda$ is not contained in $\omega$.

1.3. The restriction map as a $C_{r}$-equivariant stable map and the Bökstedt-Hsiang-Madsen conjecture. In this section we fix a finite cyclic group $C_{r}$ and identify the combinatorial restriction maps

$$
\operatorname{Res}_{G}^{H}(\lambda, \omega): \mathbb{E} \Sigma_{C_{r}}^{\infty}\left(B^{\mathrm{cy}}(G, \omega)_{+}\right) \rightarrow \mathbb{E} \Sigma_{C_{r}}^{\infty}\left(B^{\mathrm{cy}}(H, \lambda)_{+}\right)
$$

as maps in the $C_{r}$-equivariant stable homotopy category. Here $\mathbb{E} \Sigma_{C_{r}}^{\infty}(-)$ denotes the $C_{r}$-spectrum obtained from $\mathbb{E} \Sigma_{\mathbb{T}}^{\infty}(-)$ by restricting the action. The $C_{r}$-equivariant homotopy type of $B^{\mathrm{cy}}(G, \omega)$ has been determined by Lydakis [17]. We need some background material before stating the result. Consider an extension of discrete groups

$$
1 \rightarrow \Pi \rightarrow \Upsilon \rightarrow C \rightarrow 1 \text {. }
$$

A principal $(\Pi ; \Upsilon)$-bundle is a principal $\Pi$-bundle of the form $E \rightarrow E / \Pi$, where $E$ is a $\Pi$-free right $\Upsilon$-space. We say that such a principal $(\Pi ; \Upsilon)$ bundle is universal if $E$ is a $\Upsilon$-CW complex and $E^{K}$ is contractible for all subgroups $K \subseteq \Upsilon$ that satisfy $K \cap \Pi=1$. In this case $E$ is a terminal object in the homotopy category of $\Pi$-free $\Upsilon$-CW complexes, and in particular the $C$-equivariant homotopy type of $B=E / \Pi$ is uniquely determined. We say that $B$ is a classifying space for principal $(\Pi ; \Upsilon)$-bundles. The relevance of this with respect to $B^{\text {cy }}(G, \omega)$ is as follows. The choice of a representative $x \in \omega$ determines a homeomorphism

$$
\Phi(x): B^{\text {cy }}(G, \omega) \stackrel{\sim}{\rightarrow} E G / C_{G}(x),
$$

where $C_{G}(x)$ denotes the centralizer of $x$ in $G$. Thus we may view $B^{\text {cy }}(G, \omega)$ as a model of the classifying space $B C_{G}(x)$ equipped with a certain $\mathbb{T}$ action and in particular a $C_{r}$-action. We construct an extension of $C_{G}(x)$ 
by adjoining an extra element $\zeta$ and imposing the relations that $\zeta$ be central and $\zeta^{r}=x$. There results an exact sequence

$$
1 \rightarrow C_{G}(x) \rightarrow C_{G}(x)\left\{x^{1 / r}\right\} \rightarrow C_{r} \rightarrow 1,
$$

which is split if and only if $C_{G}(x)$ already contains an element $\rho$ with $\rho^{r}=x$. If $\rho$ itself is central, then $C_{G}(x)\left\{x^{1 / r}\right\} \cong C_{G}(x) \times C_{r}$.

Theorem 1.3 ([17]). The choice of a representative $x \in \omega$ makes the $C_{r}$-space $B^{c y}(G, \omega)$ a classifying space for principal $\left(C_{G}(x) ; C_{G}(x)\left\{x^{1 / r}\right\}\right)$ bundles.

We include a short proof of this result in Section 6.1. Let now $H \subseteq G$ be a subgroup of finite index and $\lambda \subseteq \omega$ a pair of conjugacy classes as above. For each representative $x \in \lambda$, the subgroup $C_{H}(x)$ then also has finite index in $C_{G}(x)$ and we shall prove that the inclusion $B^{\text {cy }}(H, \lambda) \rightarrow B^{\text {cy }}(G, \omega)$ may be identified up to $C_{r}$-equivariant homotopy with the finite $C_{r}$-equivariant covering projection

$$
E / C_{H}(x) \rightarrow E / C_{G}(x),
$$

where $E$ denotes a universal $C_{G}(x)$-free $C_{G}(x)\left\{x^{1 / r}\right\}$-CW complex as above. Let $\operatorname{Trf}_{C_{r}}$ be the associated stable transfer in the $C_{r}$-equivariant stable homotopy category. We recall the relevant definitions in Section 5.2.

Theorem 1.4. Given a pair of conjugacy classes $\omega \in\langle G\rangle$ and $\lambda \in\langle H\rangle$ with $\lambda \subseteq \omega$ and a representative $x \in \lambda$, the restriction map $\operatorname{Res}_{G}^{H}(\lambda, \omega)$ may be identified with the equivariant stable transfer in the sense that there is a commutative diagram in the $C_{r}$-equivariant stable homotopy category

$$
\begin{array}{ccc}
\mathbb{E} \Sigma_{C_{r}}^{\infty}\left(B^{c y}(G, \omega)_{+}\right) & \stackrel{\operatorname{Res}_{G}^{H}(\lambda, \omega)}{\longrightarrow} & \mathbb{E} \Sigma_{C_{r}}^{\infty}\left(B^{c y}(H, \lambda)_{+}\right) \\
\downarrow & \downarrow \sim \\
\Sigma_{C_{r}}^{\infty}\left(E / C_{G}(x)_{+}\right) & \stackrel{\operatorname{Trf}_{C_{r}}}{\longrightarrow} & \Sigma_{C_{r}}^{\infty}\left(E / C_{H}(x)_{+}\right)
\end{array}
$$

in which the vertical maps are equivalences.

Letting $x=1_{H}$, this theorem verifies the Bökstedt-Hsiang-Madsen Conjecture $[8,7.14]$, which predicts that the composition

$$
\Sigma_{C_{r}}^{\infty}\left(B G_{+}\right) \rightarrow \mathrm{TH}(\mathbb{S}[G]) \stackrel{\operatorname{Res}_{G}^{H}}{\rightarrow} \mathrm{TH}(\mathbb{S}[H]) \rightarrow \Sigma_{C_{r}}^{\infty}\left(B H_{+}\right)
$$

may be identified with the equivariant stable transfer. This implies that the transfer map in Waldhausen's algebraic K-theory of spaces is related to the equivariant stable transfer via the cyclotomic trace.

1.4. Laurent polynomials and TC transfer invariant elements. We define the Laurent polynomial ring spectrum to be the group ring spectrum $R\left[z, z^{-1}\right]=R[C]$ of the infinite cyclic group $C$. As an application of the general theory, we analyze the effect of the TC-transfer maps

$$
t_{n}: \operatorname{TC}\left(R\left[z, z^{-1}\right]\right) \rightarrow \operatorname{TC}\left(R\left[z, z^{-1}\right]\right)
$$


associated to the inclusions $z \mapsto z^{n}$ and we calculate the subgroup of transfer invariant elements

$$
\pi_{*} \mathrm{TC}\left(R\left[z, z^{-1}\right]\right)^{\mathrm{INV}}=\left\{x \in \pi_{*} \mathrm{TC}\left(R\left[z, z^{-1}\right]\right): t_{n}(x)=x \text { for all } n\right\} .
$$

Here and in the following we use the term transfer map rather than restriction map in connection with Laurent polynomial rings. More generally, we define the Laurent polynomial ring spectrum in $m$ variables to be the group ring spectrum of the $m$-fold product of $C$ and define

$$
\pi_{*} \mathrm{TC}\left(R\left[z_{1}, z_{1}^{-1}, \ldots, z_{m}, z_{m}^{-1}\right]\right)^{\mathrm{INV}}
$$

and the K-theoretical analogue

$$
\pi_{*} \mathrm{~K}\left(R\left[z_{1}, z_{1}^{-1}, \ldots, z_{m}, z_{m}^{-1}\right]\right)^{\mathrm{INV}}
$$

to be the subgroups of elements fixed by the transfers associated to the inclusions $z_{i} \mapsto z_{i}^{n}$. In the case of a discrete ring $R$, it follows from $[25,12.4]$ that the K-theoretical transfer invariants of the usual Whitehead K-group are related to the lower K-groups by an isomorphism

$$
\mathrm{K}_{1-m}(R) \cong \mathrm{K}_{1}\left(R\left[z_{1}, z_{1}^{-1}, \ldots, z_{m}, z_{m}^{-1}\right]\right)^{\mathrm{INV}}
$$

for $m \geq 1$. For $m=1$, we understand the group $\mathrm{K}_{0}(R)$ on the left hand side to mean the algebraic K-theory of finitely generated projective $R$-modules. In the geometric applications $R$ is the integral group ring associated to the fundamental group of a space $X$ and $R\left[z, z^{-1}\right]=\mathbb{Z}\left[\pi_{1}(X \times \mathbb{T})\right]$. In this case the above isomorphism is geometrically significant as it gives a geometric interpretation of the lower K-groups, see $[25, \S 12]$.

Consider now the case of the Whitehead K-group $\mathrm{K}_{1}\left(R\left[z, z^{-1}\right]\right)$ when $R$ is a discrete ring. The pairing

$$
\mathbb{Z}\left[z, z^{-1}\right] \otimes R \rightarrow R\left[z, z^{-1}\right]
$$

induces a pairing in algebraic K-theory and it follows from [25, 12.4], that multiplication by the class in $\mathrm{K}_{1}\left(\mathbb{Z}\left[z, z^{-1}\right]\right)$ represented by $-z$ induces the above isomorphism

$$
-z: \mathrm{K}_{0}(R) \stackrel{\sim}{\rightarrow} \mathrm{K}_{1}\left(R\left[z, z^{-1}\right]\right)^{\mathrm{INV}} .
$$

It should be noted that the class in $\mathrm{K}_{1}\left(\mathbb{Z}\left[z, z^{-1}\right]\right)$ represented by $z$ is not transfer invariant. Since the linearization map $\mathbb{S}\left[z, z^{-1}\right] \rightarrow \mathbb{Z}\left[z, z^{-1}\right]$ induces a 2-connected map in algebraic K-theory, the unit $-z$ corresponds to a unique class in $\pi_{1} \mathrm{~K}\left(\mathbb{S}\left[z, z^{-1}\right]\right)$ and we let

$$
u \in \pi_{1} \operatorname{TC}\left(\mathbb{S}\left[z, z^{-1}\right]\right)
$$

be its image under the cyclotomic trace. (We recall that $\mathrm{K}\left(\mathbb{S}\left[z, z^{-1}\right]\right.$ ) may be identified with Waldhausen's $A(\mathbb{T}))$. Let again $R$ be a symmetric ring spectrum and consider the pairing $\mathbb{S}\left[z, z^{-1}\right] \wedge R \rightarrow R\left[z, z^{-1}\right]$. On the level of homotopy groups this induces a pairing

$$
\pi_{i} \mathrm{TC}\left(\mathbb{S}\left[z, z^{-1}\right]\right) \otimes \pi_{j} \mathrm{TC}(R) \rightarrow \pi_{i+j} \mathrm{TC}\left(R\left[z, z^{-1}\right]\right) .
$$


Theorem 1.6. Multiplication by $u$ defines an isomorphism

$$
u: \pi_{i-1} \mathrm{TC}(R) \stackrel{\sim}{\rightarrow} \pi_{i} \mathrm{TC}\left(R\left[z, z^{-1}\right]\right)^{\mathrm{INV}}
$$

for all integers $i$.

Arguing by induction, this has the following corollary.

Corollary 1.7. There are isomorphisms

$$
\pi_{i-m} \mathrm{TC}(R) \cong \pi_{i} \mathrm{TC}\left(R\left[z_{1}, z_{1}^{-1}, \ldots, z_{m}, z_{m}^{-1}\right]\right)^{\mathrm{INV}}
$$

for all integers $i$ and all $m \geq 1$.

In particular, we conclude that

$$
\pi_{0} \mathrm{TC}\left(R\left[z, z^{-1}\right]\right)^{\mathrm{INV}}=\pi_{-1} \mathrm{TC}(R)
$$

and that the group of transfer invariant elements in Corollary 1.7 vanishes for $i<m-1$ since $\mathrm{TC}(R)$ is -2 -connected. This is consistent with the definition of negative TC-groups obtained using Bass' general approach [3, $\mathrm{XII}]$ as we now explain. Given a functor $F$ from the category of rings to the category of abelian groups, Bass defines a functor $L F$ by letting $L F(R)$ be the cokernel of the third homomorphism in the complex

$$
0 \rightarrow F(R) \stackrel{\{+,-\}}{\longrightarrow} F(R[z]) \oplus F\left(R\left[z^{-1}\right]\right) \rightarrow F\left(R\left[z, z^{-1}\right]\right) \rightarrow L F(R) \rightarrow 0,
$$

where the second homomorphism is induced by the obvious inclusions. The functor $F$ is said to be contracted if the sequence is exact and there exists a natural splitting $L F(R) \rightarrow F\left(R\left[z, z^{-1}\right]\right)$. The "fundamental theorem of algebraic K-theory" is the statement that the functors $\mathrm{K}_{i}(R)$ are contracted and that $L \mathrm{~K}_{i}(R) \simeq \mathrm{K}_{i-1}(R)$, where for $i \leq 0$ this defines the negative $\mathrm{K}$ groups. Similarly, it follows from the proof of Theorem 1.6 that the functors $\pi_{i} \mathrm{TC}(R)$ are contracted, but in this case

$$
L \pi_{i} \mathrm{TC}(R) \simeq \pi_{i-1} \mathrm{TC}(R)
$$

for all $i$. Thus, the negative TC groups vanish below degree -1 also from this point of view.

1.5. Organization of the paper. In Section 2 we present some preliminary material on cyclotomic spectra and in Section 3 we recall the definition of topological Hochschild homology and topological cyclic homology. The definitions in these sections differ somewhat from those in the literature. We begin the general analysis of restriction maps in Section 4, where we introduce the combinatorial restriction map. In Section 5 we introduce the analogous notion of a combinatorial transfer map and in Section 6 we use this to prove the main theorems with the exception of Theorem 1.6, which is proved in Section 7. Finally, in Appendix A we recall the definition of the cyclotomic trace and verify that it commutes with restriction maps. By a space we understand a compactly generated weak Hausdorff topological space throughout. 


\section{Preliminaries on CyClotomic SPeCtra}

Let $\mathbb{T}$ be the circle group. Given a finite dimensional real $\mathbb{T}$-representation $V$, the one-point compactification $S^{V}$ inherits a base-point preserving $\mathbb{T}$ action and given a based $\mathbb{T}$-space $X$, we let $\Omega^{V} X$ be the space of based maps $\operatorname{Map}\left(S^{V}, X\right)$ with $\mathbb{T}$ acting by conjugation. We shall follow the usual conventions and distinguish between spectra with $\mathbb{T}$-action and genuine $\mathbb{T}$ spectra. By a (non-equivariant) spectrum $E$, we understand a sequence of based spaces $E_{n}$ together with a sequence of based maps $\sigma: S^{1} \wedge E_{n} \rightarrow E_{n+1}$. We say that $E$ is an $\Omega$-spectrum if the adjoint maps $\tilde{\sigma}: E_{n} \rightarrow \Omega E_{n+1}$ are (weak homotopy) equivalences.

2.1. Spectra with $\mathbb{T}$-action and $\mathbb{T}$-spectra. A spectrum with $\mathbb{T}$-action $E$ is a sequence of $\mathbb{T}$-spaces $E_{n}$ together with a sequence of $\mathbb{T}$-equivariant maps $S^{1} \wedge E_{n} \rightarrow E_{n+1}$, where $\mathbb{T}$ acts trivially on $S^{1}$. In order to define the $\mathbb{T}$ equivariant stable homotopy category, we need to consider spectra indexed on a complete set of finite dimensional real $\mathbb{T}$-representations. Let $\mathbb{C}(k)$ denotes the 2-dimensional real representation obtained by letting $z \in \mathbb{T}$ act on $\mathbb{C}$ via $z^{k}$ and let $\mathcal{V}$ be the set of finite dimensional real $\mathbb{T}$-representations of the form

$$
V=\mathbb{R}^{n_{0}} \oplus \mathbb{C}(1)^{n_{1}} \oplus \mathbb{C}(2)^{n_{2}} \oplus \mathbb{C}(3)^{n_{3}} \ldots,
$$

where $n_{k}$ is non-zero for only finitely many $k$. The direct sum operation gives $\mathcal{V}$ the structure of an associative and commutative monoid

$$
\oplus: \mathcal{V} \times \mathcal{V} \rightarrow \mathcal{V}, \quad(V, W) \mapsto V \oplus W,
$$

which may be identified with the free commutative monoid on a countable infinite number of generators. A $\mathbb{T}$-spectrum $E$ is a family of based $\mathbb{T}$-spaces $E(V)$ indexed on $\mathcal{V}$ together with a family of $\mathbb{T}$-equivariant structure maps

$$
\sigma_{W}^{V}: S^{V} \wedge E(W) \rightarrow E(V \oplus W) .
$$

We require that $\sigma_{W}^{0}$ is the canonical identification and that the diagram

$$
\begin{array}{ccc}
S^{U} \wedge S^{V} \wedge E(W) & \stackrel{\sim}{\longrightarrow} S^{U \oplus V} \wedge E(W) \\
\downarrow S^{U} \wedge \sigma_{W}^{V} & & \\
S^{U} \wedge E(V \oplus W) & \stackrel{\sigma_{V \oplus W}^{U}}{\longrightarrow} E(U \oplus V \oplus W)
\end{array}
$$

is commutative for each triple $U, V$ and $W$ in $\mathcal{V}$. The homeomorphism

$$
S^{U} \wedge S^{V} \stackrel{\sim}{\rightarrow} S^{U \oplus V}
$$

is specified by letting a summand $\mathbb{R}^{n_{0}}$ or $\mathbb{C}(k)^{n_{k}}$ in $U$ correspond to the first $n_{0}$ or $n_{k}$ components of the corresponding summand in $U \oplus V$ and by letting the analogous summands in $V$ correspond to the last components. We say that a $\mathbb{T}$-spectrum is an $\Omega$-spectrum if the adjoint structure maps $E(W) \rightarrow \Omega^{V} E(V \oplus W)$ are $\mathbb{T}$-equivariant equivalences, ie, if the induced 
maps of $C$ fixed-points are equivalences for each compact subgroup $C \subseteq \mathbb{T}$. Any $\mathbb{T}$-spectrum $E$ has an associated $\Omega$-spectrum $\bar{E}$ defined by

$$
\bar{E}(W)=\underset{V}{\operatorname{hocolim}} \Omega^{V} E(V \oplus W) .
$$

Here we view $\mathcal{V}$ as a directed set where $U \leq V$ if there exists $U^{\prime}$ such that $U^{\prime} \oplus U=V$, and the homotopy colimit is over the directed system

$$
\Omega^{U} E(U \oplus W) \stackrel{\Omega^{U} \tilde{\sigma}}{\rightarrow} \Omega^{U} \Omega^{U^{\prime}} E\left(U^{\prime} \oplus U \oplus W\right) \cong \Omega^{V} E(V \oplus W),
$$

where $\tilde{\sigma}$ is the adjoint of $\sigma_{U \oplus W}^{U^{\prime}}$ and the homeomorphism

$$
\Omega^{U} \Omega^{U^{\prime}} \cong \operatorname{Map}\left(S^{U^{\prime}} \wedge S^{U},-\right) \cong \operatorname{Map}\left(S^{U^{\prime} \oplus U},-\right)=\Omega^{V}
$$

is defined using the homeomorphism specified in (2.1). In more detail, we may define a $\mathbb{T}$-spectrum $\Omega^{U} E[U]$ for each $U$ in $\mathcal{V}$ by letting

$$
\Omega^{U} E[U](V)=\Omega^{U} E(U \oplus V)
$$

with structure map $\sigma_{W}^{V}$ the adjoint of the map

$$
\Omega^{U} E(U \oplus V) \stackrel{\Omega^{U} \tilde{\sigma}}{\rightarrow} \Omega^{U} \Omega^{V} E(V \oplus U \oplus W) \cong \Omega^{V} \Omega^{U} E(U \oplus V \oplus W) .
$$

Here the homeomorphism

$\Omega^{U} \Omega^{V} \cong \operatorname{Map}\left(S^{U} \wedge S^{V},-\right) \cong \operatorname{Map}\left(S^{U \oplus V},-\right) \cong \operatorname{Map}\left(S^{V} \wedge S^{U},-\right) \cong \Omega^{V} \Omega^{U}$

is defined applying the homeomorphism in (2.1) twice, using that by definition $U \oplus V=V \oplus U$. The spectrum $\bar{E}$ is then a homotopy colimit over $\mathcal{V}$ of these spectra. A map of $\mathbb{T}$-spectra $E \rightarrow E^{\prime}$ is a family of $\mathbb{T}$-equivariant based maps $E(V) \rightarrow E^{\prime}(V)$ that commute with the structure maps. Given a compact subgroup $C \subseteq \mathbb{T}$, the associated homotopy groups are defined by

$$
\pi_{i}^{C} E=\underset{V, n}{\operatorname{colim}}\left[S^{i+n} \wedge S^{V}, E\left(\mathbb{R}^{n} \oplus V\right)\right]_{C}
$$

for all integers $i$. Here $[-,-]_{C}$ denotes the set of based $C$-equivariant homotopy classes. A map of $\mathbb{T}$-spectra is an equivalence if it induces an equivalence on homotopy groups for all compact subgroups. Notice that the natural map $E \rightarrow \bar{E}$ is an equivalence in this sense. Restricting to the trivial representations $\mathbb{R}^{n}$, a $\mathbb{T}$-spectrum $E$ gives rise to a spectrum with $\mathbb{T}$-action and given a compact subgroup $C$ we may define a spectrum by evaluating the $C$ fixedpoints in each spectrum degree. In case $E$ is an equivariant $\Omega$-spectrum we let $E^{C}$ have this meaning. However, in order to have a homotopy functor on the category of all $\mathbb{T}$-spectra one should first replace $E$ by $\bar{E}$ and then evaluate the fixed point spectrum $\bar{E}^{C}$. Notice that by definition $\pi_{i}^{C} E=\pi_{i} \bar{E}^{C}$.

2.2. Cyclotomic spectra. Given a finite cyclic subgroup $C_{r} \subseteq \mathbb{T}$, we let $\rho_{r}: \mathbb{T} \rightarrow \mathbb{T} / C_{r}$ be the homeomorphism $\rho_{r}(z)=\sqrt[r]{z}$. Given a $\mathbb{T}$-space $X$ we denote by $\rho_{r}^{*} X^{C_{r}}$ the $\mathbb{T}$-space obtained by pulling back the natural $\mathbb{T} / C_{r^{-}}$ action on $X^{C_{r}}$ via $\rho_{r}$. Notice that $\rho_{r}^{*} \mathbb{C}(k)^{C_{r}}=\mathbb{C}(k / r)$ if $r$ divides $k$ and equals $\{0\}$ otherwise. In this way we shall view $\rho_{r}^{*}(-)^{C_{r}}$ as defining a monoid homomorphism $\mathcal{V} \rightarrow \mathcal{V}$. 
Definition 2.2. A cyclotomic spectrum $E$ is a $\mathbb{T}$-spectrum equipped with a family of $\mathbb{T}$-equivariant maps

$$
R_{r}(V): \rho_{r}^{*} E(V)^{C_{r}} \rightarrow E\left(\rho_{r}^{*} V^{C_{r}}\right)
$$

satisfying the following conditions.

(i): For each pair $V$ and $W$ in $\mathcal{V}$, the diagram

$$
\begin{aligned}
& \rho_{r}^{*}\left(S^{V} \wedge E(W)\right)^{C_{r}} \stackrel{\operatorname{id} \wedge R_{r}(W)}{\longrightarrow} S_{r}^{\rho_{r}^{*} V^{C_{r}}} \wedge E\left(\rho_{r}^{*} W^{C_{r}}\right) \\
& \downarrow \rho_{r}^{*}\left(\sigma_{W}^{V}\right)^{C_{r}} \quad \downarrow \sigma_{\rho_{r}^{*} W^{*} C_{r}}^{\rho_{r}^{*} C_{r}} \\
& \rho_{r}^{*} E(V \oplus W)^{C_{r}} \stackrel{R_{r}(V \oplus W)}{\longrightarrow} E\left(\rho_{r}^{*}(V \oplus W)^{C_{r}}\right)
\end{aligned}
$$

is commutative.

(ii): For each $V$ in $\mathcal{V}$ and each pair of cyclic groups $C_{r}$ and $C_{s}$, the diagram

$$
\begin{array}{cr}
\rho_{r}^{*}\left(\rho_{s}^{*} E(V)^{C_{s}}\right)^{C_{r}} & \rho_{r s}^{*} E(V)^{C_{r s}} \\
\rho_{r}^{*} R_{s}(V)^{C_{r}} & \downarrow R_{r s}(V) \\
\rho_{r}^{*} E\left(\rho_{s}^{*} V^{C_{s}}\right)^{C_{r}} \stackrel{R_{r}\left(\rho_{s}^{*} V^{C_{s}}\right)}{\longrightarrow} E\left(\rho_{r s}^{*} V^{C_{r s}}\right) .
\end{array}
$$

is commutative.

Remark 2.3. The notion of a cyclotomic spectrum used here is an adaptation of the notion of a cyclotomic prespectrum introduced in Section 2 of [14], except that we omit condition (iii) in the definition given there. This condition is necessary in order to establish the fundamental cofibration sequence of a cyclotomic spectrum [14, 2.2], but we find it useful to have this more general definition. The analogous notion of a cyclotomic space is considered in Appendix A.1.

A map of cyclotomic spectra $E \rightarrow E^{\prime}$ is a map of $\mathbb{T}$-spectra whose components $E(V) \rightarrow E^{\prime}(V)$ commute with the cyclotomic structure maps. When considering cyclotomic spectra we are usually only interested in the fixedpoint spectra for finite cyclic groups. Thus we say that a map of cyclotomic spectra is an equivalence if it induces an equivalence on the homotopy groups $\pi_{*}^{C_{r}}$ for each finite cyclic group (but not necessarily for $\mathbb{T}$ itself). If $E$ and $E^{\prime}$ are equivariant $\Omega$-spectra, this is equivalent to the induced map of $C_{r}$ fixedpoint spectra being equivalences for all $r$. We say that $E$ is a cyclotomic $\Omega$-spectrum if the adjoint structure maps are $C_{r}$-equivariant equivalences for all $r$. This is weaker than being an $\Omega \mathbb{T}$-spectrum.

Lemma 2.4. A cyclotomic structure on $E$ induces a cyclotomic structure on the associated $\Omega \mathbb{T}$-spectrum $\bar{E}$ such that the natural map $E \rightarrow \bar{E}$ is an equivalence of cyclotomic spectra.

Proof. We define the cyclotomic structure map

$$
R_{r}(W): \rho_{r}^{*} \bar{E}(W)^{C_{r}} \rightarrow \bar{E}\left(\rho_{r}^{*} W^{C_{r}}\right)
$$


to be the composition of (i) the map of homotopy colimits induced by the natural transformations

$$
\rho_{r}^{*} \Omega^{V} E(V \oplus W)^{C_{r}} \rightarrow \Omega^{\rho_{r}^{*} V^{C_{r}}} \rho_{r}^{*} E(V \oplus W)^{C_{r}} \rightarrow \Omega^{\rho_{r}^{*} V^{C_{r}}} E\left(\rho_{r}^{*} V^{C_{r}} \oplus \rho_{r}^{*} W^{C_{r}}\right)
$$

given by $f \mapsto R_{r}(V \oplus W) \circ f^{C_{r}}$, and (ii) the map

$$
\underset{V}{\operatorname{hocolim}} \Omega^{\rho_{r}^{*} V^{C_{r}}} E\left(\rho_{r}^{*} V^{C_{r}} \oplus \rho_{r}^{*} W^{C_{r}}\right) \rightarrow \underset{V}{\operatorname{hocolim}} \Omega^{V} E\left(V \oplus \rho_{r}^{*} W^{C_{r}}\right)
$$

induced by the monoid homomorphism $\rho_{r}^{*}(-)^{C_{r}}: \mathcal{V} \rightarrow \mathcal{V}$ thought of as a map of directed sets. With this definition it is clear that $E \rightarrow \bar{E}$ is a map of cyclotomic spectra.

Suppose now that $E$ is a cyclotomic $\Omega$-spectrum such that the level-wise fixed-point spectra $E^{C_{r}}$ of the underlying spectrum with $\mathbb{T}$-action have the correct homotopy type. The cyclotomic structure maps restrict to maps of spectra with $\mathbb{T}$-action $R_{r}: \rho_{r}^{*} E^{C_{r}} \rightarrow E$, which in turn give rise to maps

$$
R_{s}: \rho_{r s}^{*} E^{C_{r s}}=\rho_{r}^{*}\left(\rho_{s}^{*} E^{C_{s}}\right)^{C_{r}} \stackrel{\rho_{r}^{*}\left(R_{s}\right)^{C_{r}}}{\longrightarrow} \rho_{r}^{*} E^{C_{r}} .
$$

Let $\mathbb{I}$ be the category whose objects are the natural numbers $1,2, \ldots$, and whose morphisms are generated by two types of arrows $R_{s}, F_{s}: r s \rightarrow r$, subject to the relations

$$
R_{1}=F_{1}=\mathrm{id}, \quad R_{r} R_{s}=R_{r s}, \quad F_{r} F_{s}=F_{r s}, \quad R_{r} F_{s}=F_{s} R_{r} .
$$

Any morphism in $\mathbb{I}$ can thus be written uniquely in the form $F_{s} R_{r}$. We call the $F_{s}$ arrows Frobenius morphisms and the $R_{s}$ arrows restriction morphisms. A cyclotomic $\Omega$-spectrum $E$ gives rise to an $\mathbb{I}$-diagram of (nonequivariant) $\Omega$-spectra

$$
R_{s}, F_{s}: E^{C_{r s}} \rightarrow E^{C_{r}}
$$

in which the action of the restriction morphisms are given by the maps in (2.5) (ignoring that they are $\mathbb{T}$-equivariant) and the Frobenius morphisms act via the natural fixed-point inclusions.

2.3. Smash products and bispectra. Given $\mathbb{T}$-spectra $E$ and $E^{\prime}$, the levelwise smash products $E(V) \wedge E^{\prime}(W)$ naturally form a $\mathbb{T}$-bispectrum indexed on $\mathcal{V} \times \mathcal{V}$. We define the associated $\Omega \mathbb{T}$-bispectrum $E \wedge \mathcal{V} E^{\prime}$ by letting

$$
E \wedge \mathcal{V} E^{\prime}\left(W, W^{\prime}\right)=\underset{V, V^{\prime}}{\operatorname{hocolim}} \Omega^{V} \Omega^{V^{\prime}}\left(E(V \oplus W) \wedge E^{\prime}\left(V^{\prime} \oplus W^{\prime}\right)\right),
$$

where structure maps similar to those of $\bar{E}$. Notice that if $E$ and $E^{\prime}$ are cyclotomic spectra, then $E \wedge \mathcal{V} E^{\prime}$ inherits the structure of a cyclotomic bispectrum by an argument similar to that of Lemma 2.4. For the rest of the paper we fix a functor from $\mathbb{T}$-bispectra indexed on $\mathcal{V} \times \mathcal{V}$ to $\mathbb{T}$ spectra along the lines of the handicrafted smash products of $[1,4]$. Given a representation $V$ in the standard form with summands $\mathbb{R}^{n_{0}}$ and $\mathbb{C}(k)^{n_{k}}$, we let

$$
n_{i}^{\prime}=\left\{\begin{array}{ll}
m_{i}, & \text { if } n_{i}=2 m_{i} \\
m_{i}+1, & \text { if } n_{i}=2 m+1
\end{array}, \quad n_{i}^{\prime \prime}= \begin{cases}m_{i}, & \text { if } n_{i}=2 m_{i} \\
m_{i}, & \text { if } n_{i}=2 m+1\end{cases}\right.
$$


and define $V^{\prime}$ and $V^{\prime \prime}$ to have summands $\mathbb{R}^{n_{0}^{\prime}}$ and $\mathbb{C}(k)^{n_{k}^{\prime}}$, respectively $\mathbb{R}^{n_{0}^{\prime \prime}}$ and $\mathbb{C}(k)^{n_{k}^{\prime \prime}}$. Then $V^{\prime} \oplus V^{\prime \prime}=V$ and if $E$ is a $\mathbb{T}$-bispectrum we define the associated $\mathbb{T}$-spectrum $\delta E$ by letting

$$
\delta E(V)=E\left(V^{\prime}, V^{\prime \prime}\right) .
$$

This has a canonical structure of a $\mathbb{T}$-spectrum with structure maps

$$
S^{V} \wedge \delta E(W) \rightarrow \delta E(V \oplus W) .
$$

Indeed, there is an obvious choice of such maps when $V$ has the form $\mathbb{R}$ or $\mathbb{C}(k)$ and these choices extend uniquely to give a $\mathbb{T}$-spectrum. Furthermore, if $E$ is a cyclotomic bispectrum, then $\delta E$ inherits the structure of a cyclotomic spectrum. We usually omit the symbol $\delta$ and talk about the underlying diagonal $\mathbb{T}$-spectrum of a $\mathbb{T}$-bispectrum.

\section{TOPOLOGICAL CYCLIC HOMOLOGY}

Let $R$ be a symmetric ring spectrum in the category of spaces as defined in [22]. We shall always assume that $R$ is (i) connective in the sense that $\pi_{i} R=0$ for $i<0$ and (ii) convergent in the sense that there exists an unbounded, non-decreasing sequence of integers $\lambda_{n}$ such that the adjoint structure maps $E_{n} \rightarrow \Omega E_{n+1}$ are $n+\lambda_{n}$-connected.

3.1. Topological Hochschild homology. In this section we apply a construction similar to that of Dundas-McCarthy [11] to define the topological Hochschild homology and the topological cyclic homology of the category $\mathcal{F}(R)$ of finitely generated free $R$-modules. Let $\mathcal{F}$ be the skeleton category of finite based sets. For definiteness we specify the objects of $\mathcal{F}(R)$ to be ordered wedge products of the form $\vee_{i=1}^{r} S^{0}$. The empty wedge product $*$ makes $\mathcal{F}$ a based category. Let $\mathcal{F}(R)$ be the set of symmetric spectra of the form $R(S)=R \wedge S$ where $S$ is an object in $\mathcal{F}$. This may of course be identified with the ordered wedge products $R^{\vee r}$. Given objects $S$ and $S^{\prime}$ in $\mathcal{F}$, we define $M\left(R(S), R\left(S^{\prime}\right)\right)$ to be the symmetric spectrum whose $n$th space is given by

$$
M\left(R(S), R\left(S^{\prime}\right)\right)_{n}=\operatorname{Map}\left(S, R_{n} \wedge S^{\prime}\right) .
$$

There are natural associative pairings of symmetric spectra

$$
M\left(R\left(S^{\prime}\right), R\left(S^{\prime \prime}\right)\right) \wedge M\left(R(S), R\left(S^{\prime}\right)\right) \rightarrow M\left(R(S), R\left(S^{\prime \prime}\right)\right)
$$

and the unit of $R$ provides each of the symmetric spectra $M(R(S), R(S))$ with a unit. We may thus view $\mathcal{F}(R)$ as a category enriched in symmetric spectra in the sense that there is a symmetric spectrum of "morphisms" relating any two objects. In particular, $M(R(S), R(S))$ is a symmetric ring spectrum and as such it is isomorphic to the usual matrix spectrum [8, 3.2]. Let $V_{k}[\mathcal{F}(R)]$ be the $(k+1)$-fold multi-symmetric spectrum defined by

$$
V_{k}[\mathcal{F}(R)]=\bigvee_{S_{0}, \ldots, S_{k}} M\left(R\left(S_{0}\right), R\left(S_{k}\right)\right) \wedge \cdots \wedge M\left(R\left(S_{k}\right), R\left(S_{k-1}\right)\right),
$$


where $S_{0}, \ldots, S_{k}$ runs through all $(k+1)$-tuples of objects in $\mathcal{F}$. Here we view the external smash product of a sequence of symmetric spectra as a multi-indexed spectrum in the natural way. Let $\mathcal{I}$ be the category whose objects are the the finite sets $\mathbf{n}=\{1, \ldots, n\}$ and whose morphisms are the injective maps. The definition of the cyclotomic topological Hochschild homology spectrum is based on Bökstedt's functor $Q_{\mathcal{I}^{k+1}}$ that to a $(k+1)$ fold multi-symmetric spectrum

$$
E=\left\{E\left\langle n_{0}, \ldots, n_{k}\right\rangle: n_{0}, \ldots, n_{k} \geq 0\right\}
$$

associates the homotopy colimit

$$
Q_{\mathcal{I}^{k+1}}(E)=\underset{\mathcal{I}^{k+1}}{\operatorname{hocolim}} \operatorname{Map}\left(S^{n_{0}} \wedge \cdots \wedge S^{n_{k}}, E\left\langle n_{0}, \ldots, n_{k}\right\rangle\right) .
$$

The structure maps of the $\mathcal{I}^{k+1}$-diagram on the right side are defined as in $[8,3]$. Given a representation $V$ in $\mathcal{V}$, we let $\operatorname{TH}(R, V)$ be the realization of the cyclic space

$$
\mathrm{TH}_{\bullet}(R, V):[k] \mapsto Q_{\mathcal{I}^{k+1}}\left(V_{k}[\mathcal{F}(R)] \wedge S^{V}\right)
$$

with cyclic structure defined as in $[11,1.3]$. This has an action of $\mathbb{T} \times \mathbb{T}$ with one factor acting on $S^{V}$ and the action of the other factor coming from the cyclic structure. We view $\mathrm{TH}(R, V)$ as a $\mathbb{T}$-space with the diagonal action and let $\mathrm{TH}(R)$ be the resulting $\mathbb{T}$-spectrum equipped with the natural structure maps

$$
S^{V} \wedge \mathrm{TH}(R, W) \rightarrow \mathrm{TH}(R, V \oplus W) .
$$

This has the structure of a cyclotomic spectrum with structure maps

$$
R_{r}(V): \rho_{r}^{*} \mathrm{TH}(R, V)^{C_{r}} \rightarrow \mathrm{TH}\left(R, \rho_{r}^{*} V^{C_{r}}\right)
$$

defined as in $[14,2.5]$. It follows by an argument similar to that in the proof of $[14,2.4]$ that this is a cyclotomic $\Omega$-spectrum. We use the same notation $\mathrm{TH}(R)$ for the underlying spectrum with $\mathbb{T}$-action; the meaning will always be clear from the context. As for any cyclotomic spectrum there is an associated $\mathbb{I}$-diagram

$$
F_{s}, R_{s}: \mathrm{TH}(R)^{C_{r s}} \rightarrow \mathrm{TH}(R)^{C_{r}}
$$

and by definition the topological cyclic homology spectrum is the corresponding homotopy limit

$$
\mathrm{TC}(R)=\underset{\mathbb{I}}{\operatorname{holim}} \mathrm{TH}(R)^{C_{r}} .
$$

Remark 3.1. It follows by an argument similar to that of Dundas-McCarthy $[11,2.1 .5]$ that this definition of $\mathrm{TC}(R)$ is equivalent to the original definition in [8]. More precisely, there is a natural map from the topological Hochschild homology spectrum defined there to our definition which is an equivalence of cyclotomic spectra. The approach taken here has the advantage that we may define restriction maps on the level of categories as is usually done for algebraic K-theory, see Section 4 and Appendix A. 
3.2. Pairings in topological Hochschild homology. In this section we consider the TH- and TC-pairings induced by a multiplicative pairing of symmetric ring spectra $R \wedge R^{\prime} \rightarrow R^{\prime \prime}$. Recall from [22] that such a pairing amounts to a family of $\Sigma_{m} \times \Sigma_{n}$-equivariant maps $R_{m} \times R_{n}^{\prime} \rightarrow R_{m+n}^{\prime \prime}$ satisfying certain compatibility conditions. There is an induced pairing of enriched categories

$$
\mathcal{F}(R) \times \mathcal{F}\left(R^{\prime}\right) \rightarrow \mathcal{F}\left(R^{\prime \prime}\right), \quad\left(R(S), R^{\prime}\left(S^{\prime}\right)\right) \mapsto R^{\prime \prime}\left(S \wedge S^{\prime}\right),
$$

where, given $S=\vee_{i=1}^{r} S^{0}$ and $S^{\prime}=\vee_{j=1}^{r^{\prime}} S^{0}$, we identify $S \wedge S^{\prime}$ with an object in $\mathcal{F}$ by lexicographically ordering the wedge summands. This in turn gives rise to pairings of $(k+1)$-fold multi-symmetric spectra

$$
V_{k}[\mathcal{F}(R)] \wedge V_{k}\left[\mathcal{F}\left(R^{\prime}\right)\right] \rightarrow V_{k}\left[\mathcal{F}\left(R^{\prime \prime}\right)\right]
$$

for all $k \geq 0$. In order to get a pairing in topological Hochschild homology we consider the modified version of Bökstedt's functor defined by

$Q_{\mathcal{I}^{k+1}}^{\prime}(E)=\operatorname{hocolim}_{\mathcal{I}^{k+1} \times \mathcal{I}^{k+1}} \operatorname{Map}\left(S^{m_{0}+n_{0}} \wedge \cdots \wedge S^{m_{k}+n_{k}}, E\left\langle m_{0}+n_{0}, \ldots, m_{k}+n_{k}\right\rangle\right)$

and let $\mathrm{TH}^{\prime}\left(R^{\prime \prime}\right)$ be the corresponding modified version of $\mathrm{TH}\left(R^{\prime \prime}\right)$. It follows from Bökstedt's approximation lemma $[21,2.3 .6]$ that the diagonal inclusion of $\mathcal{I}^{k+1}$ induces an equivalence of cyclotomic spectra $\mathrm{TH}\left(R^{\prime \prime}\right) \rightarrow \mathrm{TH}^{\prime}\left(R^{\prime \prime}\right)$. For each pair $V$ and $W$ in $\mathcal{V}$, the above pairings of multi-symmetric spectra give rise to a $\mathbb{T}$-equivariant map

$$
\mathrm{TH}(R, V) \wedge \mathrm{TH}\left(R^{\prime}, W\right) \rightarrow \mathrm{TH}^{\prime}\left(R^{\prime \prime}, V \oplus W\right),
$$

which we may view as the components of a map of cyclotomic bispectra. Passing to homotopy colimits as in Section 2.3, we get a chain of maps of cyclotomic $\Omega$-bispectra

$$
\mathrm{TH}(R) \wedge \mathcal{V} \mathrm{TH}\left(R^{\prime}\right) \rightarrow \overline{\mathrm{TH}}^{\prime}\left(R^{\prime \prime}\right) \stackrel{\mathrm{TH}}{ }\left(R^{\prime \prime}\right) \simeq \mathrm{TH}\left(R^{\prime \prime}\right),
$$

where $\overline{\mathrm{TH}}$ means the homotopy colimit construction in Section 2.1. The last map is an equivalence since $\operatorname{TH}\left(R^{\prime \prime}\right)$ is already a cyclotomic $\Omega$-spectrum. Restricting to the underlying diagonal $\mathbb{T}$-spectra as in Section 2.3 we view this as a chain of maps of cyclotomic spectra. We say that the pairing $R \wedge R^{\prime} \rightarrow R^{\prime \prime}$ is an equivalence if the associated map of bispectra induces an isomorphism on homotopy groups, that is, if

$$
\underset{m, n}{\operatorname{colim}} \pi_{i+m+n}\left(R_{m} \wedge R_{n}^{\prime}\right) \stackrel{\sim}{\rightarrow} \operatorname{colim}_{m, n} \pi_{i+m+n}\left(R_{m+n}^{\prime \prime}\right) \cong \pi_{i}\left(R^{\prime \prime}\right)
$$

is an isomorphism for each $i$. Using elementary equivariant homotopy theory along the lines of $[8,3.10-3.12]$, one proves the following.

Proposition 3.2. If the multiplicative pairing $R \wedge R^{\prime} \rightarrow R^{\prime \prime}$ is an equivalence, then the above chain of maps define an equivalence of cyclotomic spectra

$$
\mathrm{TH}(R) \wedge_{\mathcal{V}} \mathrm{TH}\left(R^{\prime}\right) \simeq \mathrm{TH}\left(R^{\prime \prime}\right)
$$




\section{RESTRICTION MAPS IN TOPOLOGICAL CYCLIC HOMOLOGY}

By a based monoid $\Pi$ we understand a discrete monoid equipped with a base point such that the multiplication factors over the smash product to give a based map $\Pi \wedge \Pi \rightarrow \Pi$. Given a symmetric ring spectrum $R$, the associated symmetric monoid ring spectrum is defined by $R(\Pi)=R \wedge \Pi$. Adding a disjoint base point to a discrete group (or monoid) $G$ we get a based monoid $G_{+}$and $R\left(G_{+}\right)$may be identified with the symmetric group ring spectrum $R[G]$ considered in Section 1. In order to give an example not of this form, let $\Pi_{n}$ be the based monoid of monomials in the truncated polynomial ring $\mathbb{Z}[x] /\left(x^{n}\right)$ as in $[14,7.1]$. Then $R\left(\Pi_{n}\right)$ is the truncated polynomial ring spectrum with coefficients in $R$.

4.1. Restriction functors. In general, a homomorphism of based monoids $f: \Pi \rightarrow \Upsilon$ induces a map of symmetric ring spectra $R(\Pi) \rightarrow R(\Upsilon)$ and thus a map in topological Hochschild homology

$$
f_{*}: \mathrm{TH}(R(\Pi)) \rightarrow \mathrm{TH}(R(\Upsilon)) .
$$

Suppose now that $\Upsilon$ is free of rank $n$ as a right $\Pi$-set via $f$ and that we have specified an isomorphism $\phi: \Pi^{\vee n} \stackrel{\sim}{\rightarrow} \Upsilon$ of right $\Pi$-sets. In this case we may and shall always assume that the restriction of $\phi$ to the first wedge component equals $f$. These data determine a restriction functor of enriched categories

$$
f^{*}: \mathcal{F}(R(\Upsilon)) \rightarrow \mathcal{F}(R(\Pi))
$$

by using the isomorphism of right $R(\Pi)$-module spectra determined by $\phi$,

$$
R(\Pi)\left(\vee_{i=1}^{n} S^{0} \wedge S\right) \cong R \wedge \Pi^{\vee n} \wedge S \stackrel{\phi}{\rightarrow} R \wedge \Upsilon \wedge S=R(\Upsilon)(S)
$$

and identifying the domain with an object of $\mathcal{F}(R(\Pi))$ by reverse lexicographical ordering of the wedge summands. The last step amounts to forming an ordered wedge of copies of $\vee_{i=1}^{n} S^{0}$. We need to specify the isomorphism $\phi$ since we work with skeleton categories. Clearly the restriction functors associated to two such choices are related by a natural isomorphism. The restriction (or transfer) map in topological Hochschild homology is the induced map of cyclotomic spectra

$$
f^{*}: \operatorname{TH}(R(\Upsilon)) \rightarrow \mathrm{TH}(R(\Pi)) .
$$

Lemma 4.1. The TH-restriction map $f^{*}$ is independent of the choice of $\phi$ up to canonical $C_{r}$-equivariant homotopy for all $r \geq 1$.

Before we give the proof we recall that the natural notion of homotopy for cyclic sets is that of a special homotopy $\left[19\right.$, A.5]: Let $B_{\bullet}^{\text {cy }}(J)$ be the cyclic bar construction $[19,2.2 .3]$ of the groupoid $J$ with two objects 0 and 1 and two non-identity morphisms $0 \rightarrow 1$ and $1 \rightarrow 0$. Two cyclic maps $f_{\bullet}, g_{\bullet}: X_{\bullet} \rightarrow Y_{\bullet}$ are said to be specially homotopic if there exists a cyclic map

$$
X_{\bullet} \times B_{\bullet}^{\mathrm{cy}}(J) \rightarrow Y_{\bullet}
$$


such that the restrictions to $X_{\bullet} \times\left\{\operatorname{id}_{0}\right\}$ and $X_{\bullet} \times\left\{\operatorname{id}_{1}\right\}$ equals $f_{\bullet}$ and $g \bullet$ respectively. It is proved in [19, A.4.2] that the $C_{r}$ fixed-point sets of the topological realization $B^{\text {cy }}(J)$ are contractible for all $r$ and that the $\mathbb{T}$ fixedpoint set consists of the vertices $\left\{\operatorname{id}_{0}, \mathrm{id}_{1}\right\}$. It follows that the realizations $f$ and $g$ are $C_{r}$-equivariantly homotopic for all $r$. With the notation of $[8,1]$, we get a canonical homotopy by using the composition

$$
\left|\Delta_{\bullet}[1]\right| \rightarrow\left|B_{\bullet}^{\mathrm{cy}}(J)\right| \stackrel{\Delta_{r}}{\rightarrow}\left|\operatorname{sd}_{r} B_{\bullet}^{\mathrm{cy}}(J)^{C_{r}}\right| \stackrel{D_{r}^{C_{r}}}{\rightarrow} B^{\mathrm{cy}}(J)^{C_{r}},
$$

where the first map is the realization of the simplicial map determined by $0 \mapsto \mathrm{id}_{0}$ and $1 \mapsto \mathrm{id}_{1}$.

Proof of Lemma 4.1. As remarked above, the restriction functors associated to two choices of isomorphism $\Pi^{\vee n} \rightarrow \Upsilon$ are related by a natural isomorphism. By an argument similar to that used in the linear case in [19, 2.4.1], it follows that such a natural isomorphism give rise to special homotopies

$$
\mathrm{TH}_{\bullet}(R(\Upsilon), V) \times B_{\bullet}^{\mathrm{cy}}(J) \rightarrow \mathrm{TH}_{\bullet}(R(\Pi), V)
$$

for each representations $V$. The conclusion then follows from the above remarks.

Passing to fixed-point spectra and evaluating the homotopy limit over $\mathbb{I}$, we get the restriction map in topological cyclic homology

$$
f^{*}: \mathrm{TC}(R(\Upsilon)) \rightarrow \mathrm{TC}(R(\Pi)) .
$$

It follows from Lemma 4.1 and its proof that this is well-defined up to canonical homotopy. Let now $\mathbb{S}$ be the sphere spectrum and consider the multiplicative pairing of symmetric ring spectra $\mathbb{S}(\Pi) \wedge R \rightarrow R(\Pi)$. This is an equivalence in the sense of Section 3.2 and by Proposition 3.2 it induces an equivalence of cyclotomic spectra

$$
\mathrm{TH}(\mathbb{S}(\Pi)) \wedge_{\mathcal{V}} \mathrm{TH}(R) \simeq \mathrm{TH}(R(\Pi)) .
$$

It follows immediately from the definitions that this equivalence is compatible with restriction maps in the sense of the following proposition.

Proposition 4.2. There is a diagram of cyclotomic spectra

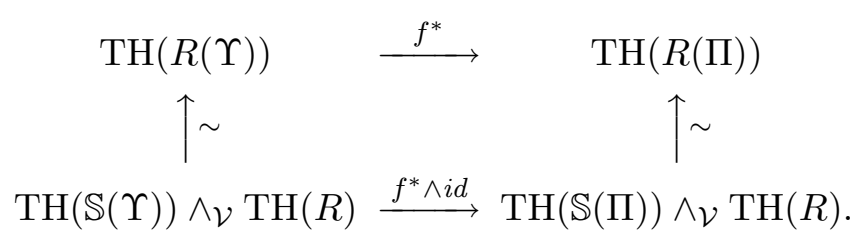

in which the vertical arrows represent chains of equivalences each of which commute with the induced restriction map $f^{*}$.

Thus it suffices to analyze the restriction maps in the case $R=\mathbb{S}$. In order to do this we shall introduce the combinatorial restriction map

$$
f^{*}: \mathbb{E} \Sigma_{\mathbb{T}}^{\infty}\left(B^{\mathrm{cy}}(\Upsilon)_{+}\right) \rightarrow \mathbb{E} \Sigma_{\mathbb{T}}^{\infty}\left(B^{\mathrm{cy}}(\Pi)_{+}\right),
$$


where $B^{\mathrm{cy}}$ is the based version of Waldhausen's cyclic bar construction and $\mathbb{E} \Sigma_{\mathbb{T}}^{\infty}$ is a cyclotomic version of the combinatorial Barratt-Eccles model of the suspension spectrum. This is a map of cyclotomic spectra and provides a model of the TH-restriction map in the following sense.

Proposition 4.3. There is a diagram of cyclotomic spectra

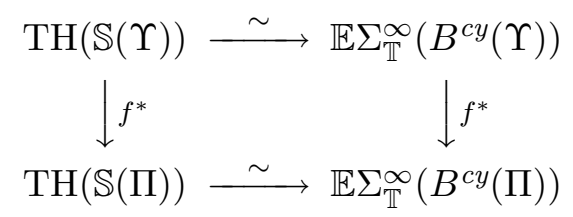

in which the horizontal arrows represent chains of equivalences of cyclotomic spectra, each of which commutes strictly with the induced restriction maps.

The proof will be given in Section 4.3 after having introduced the relevant cyclotomic spectra in Section 4.2. In Section 6 we shall analyze the combinatorial restriction map in the group ring case and use this to prove the theorems in Section 1. The analysis in [5] of Bloch's curves on algebraic Ktheory is based on the explicit definition of the combinatorial restriction map in the case $f: \Pi_{m} \rightarrow \Pi_{m n}$ is an inclusion of the based monoids introduced above.

4.2. The cyclotomic Barratt-Eccles suspension spectrum. Let $E_{\bullet}$ be the functor from sets to cyclic sets defined by

$$
E_{\bullet} Z:[k] \mapsto \operatorname{Map}([k], Z)=Z^{k+1}
$$

with the usual simplicial structure and cyclic operators

$$
t_{k}\left(z_{0}, \ldots, z_{k}\right)=\left(z_{k}, z_{0}, \ldots, z_{k-1}\right) .
$$

Applied to the symmetric group $\Sigma_{n}$ this gives a cyclic set $E_{\bullet} \Sigma_{n}$ with a coordinate-wise right $\Sigma_{n}$-action. The correspondence $n \mapsto E_{\bullet} \Sigma_{n}$ defines a contravariant functor on the category $\mathcal{I}$ introduced in Section 3.1: Given a morphism $\alpha: \mathbf{m} \rightarrow \mathbf{n}$ in $\mathcal{I}$ and an element $\sigma \in \Sigma_{n}$, the composition $\sigma \circ \alpha$ has a unique factorization in the form $\sigma_{*}(\alpha) \circ \alpha^{*}(\sigma)$, where $\alpha^{*}(\sigma) \in \Sigma_{m}$ and $\sigma_{*}(\alpha): \mathbf{m} \rightarrow \mathbf{n}$ is an order preserving injective map. The action of $\alpha$ is then given by the cyclic map

$$
\alpha^{*}: E_{\bullet} \Sigma_{n} \rightarrow E_{\bullet} \Sigma_{m}, \quad\left(\sigma_{0}, \ldots, \sigma_{k}\right) \mapsto\left(\alpha^{*}\left(\sigma_{0}\right), \ldots, \alpha^{*}\left(\sigma_{k}\right)\right) .
$$

A based space $X$ gives rise to an $\mathcal{I}$-diagram $\mathbf{n} \mapsto X^{n}$ by letting the morphisms act on the coordinates in the natural way. We use the notation $\mathbb{E}_{\bullet}^{\infty}$ for the associated functor from based spaces to cyclic based spaces,

$$
\mathbb{E}_{\bullet}^{\infty}(X)=\left(\coprod_{n=0}^{\infty} E_{\bullet} \Sigma_{n} \times X^{n}\right) /\left(\alpha^{*}(e), \mathbf{x}\right) \sim\left(e, \alpha_{*}(\mathbf{x})\right) .
$$

This is a coend in the sense of $\left[18\right.$, IX.6] and is what is denoted $\Gamma^{+}(X)$ in [4]. We claim that $\mathbb{E}_{\bullet}^{\infty}(X)$ naturally has the structure of an epicyclic space in the sense of Goodwillie, see Appendix A.1. It is clear from the definition 
of the edgewise subdivision functor $\operatorname{sd}_{r}$ that $\operatorname{sd}_{r} E_{\bullet} \Sigma_{n}$ may be identified with the $r$-fold product $\left(E_{\bullet} \Sigma_{n}\right)^{r}$ with $C_{r}$ acting by cyclic permutation and consequently

$$
\operatorname{sd}_{r} \mathbb{E}_{\bullet}^{\infty}(X) \cong\left(\coprod_{n=0}^{\infty}\left(E_{\bullet} \Sigma_{n}\right)^{r} \times X^{n}\right) /\left(\alpha^{*}(e), \mathbf{x}\right) \sim\left(e, \alpha_{*}(\mathbf{x})\right),
$$

where now $e$ denotes an element in $\left(E_{\bullet} \Sigma_{n}\right)^{r}$. We must produce a compatible family of cyclic maps

$$
R_{r}: \operatorname{sd}_{r} \mathbb{E}_{\bullet}^{\infty}(X)^{C_{r}} \rightarrow \mathbb{E}_{\bullet}^{\infty}(X) .
$$

Suppose given a $C_{r}$ fixed-point of the form $\left[\left(e_{1}, \ldots, e_{r}\right) ; \mathbf{x}\right]$ with $e_{i} \in E_{k} \Sigma_{n}$ and $\mathbf{x} \in X^{n}$. Then we define

$$
R_{r}\left(\left[\left(e_{1}, \ldots, e_{r}\right) ; \mathbf{x}\right]\right)=\left[\alpha^{*}\left(e_{1}\right), \alpha^{*}(\mathbf{x})\right],
$$

where $\alpha: \mathbf{m} \rightarrow \mathbf{n}$ is any morphism in $\mathcal{I}$ whose image equals the set

$$
\left\{s \in \mathbf{n}: e_{1}(s)=\cdots=e_{r}(s)\right\} .
$$

Here each $e_{i}(s)$ denotes a $(k+1)$-tuple of elements in $\mathbf{n}$ and the element $\alpha^{*}(\mathbf{x}) \in X^{m}$ is obtained by restricting to the coordinates of $\mathbf{x}$ specified by $\alpha$. It is easy to check that these structure maps satisfy the axioms for an epicyclic space. It follows from the discussion in Appendix A.1 that the topological realization $\mathbb{E}^{\infty}(X)$ has the structure of a cyclotomic space with structure maps

$$
R_{r}: \rho_{r}^{*} \mathbb{E}^{\infty}(X)^{C_{r}} \rightarrow \mathbb{E}^{\infty}(X) .
$$

Suppose now that $X$ itself has an action of $\mathbb{T}$. Then $\mathbb{E}^{\infty}(X)$ has an action of $\mathbb{T} \times \mathbb{T}$ and we view it as a $\mathbb{T}$ space with the diagonal action. Checking from the definitions one finds that the above constructions now produce a $\mathbb{T}$-equivariant map of the form

$$
R_{r}: \rho_{r}^{*} \mathbb{E}^{\infty}(X)^{C_{r}} \rightarrow \mathbb{E}^{\infty}\left(\rho_{r}^{*} X^{C_{r}}\right) .
$$

Definition 4.5. Let $X$ be a $\mathbb{T}$-space. The $\mathbb{T}$-spectrum $\mathbb{E} \Sigma_{\mathbb{T}}^{\infty}(X)$ is defined by letting

$$
\mathbb{E} \Sigma_{\mathbb{T}}^{\infty}(X)(V)=\mathbb{E}^{\infty}\left(S^{V} \wedge X\right)
$$

with the natural structure maps. If $X$ is a cyclotomic space, then $\mathbb{E} \Sigma_{\mathbb{T}}^{\infty}(X)$ inherits the structure of a cyclotomic spectrum with structure maps

$$
R_{r}(V): \rho_{r}^{*} \mathbb{E}^{\infty}\left(S^{V} \wedge X\right)^{C_{r}} \rightarrow \mathbb{E}^{\infty}\left(S^{\rho_{r}^{*} V^{C_{r}}} \wedge \rho_{r}^{*} X^{C_{r}}\right) \rightarrow \mathbb{E}^{\infty}\left(S^{\rho_{r}^{*} V^{C_{r}}} \wedge X\right),
$$

where the first map is defined as above and the second is induced by the cyclotomic structure of $X$.

Given a $\mathbb{T}$-space $X$, the suspension spectrum $\Sigma_{\mathbb{T}}^{\infty}(X)$ is the $\mathbb{T}$-spectrum whose $V$ th space equals $S^{V} \wedge X$ with the natural structure maps. If $X$ is a cyclotomic space then this inherits the structure of a cyclotomic spectrum. 
Proposition 4.6. If $X$ is a cyclotomic space, then the natural map

$$
\Sigma_{\mathbb{T}}^{\infty}(X) \rightarrow \mathbb{E} \Sigma_{\mathbb{T}}^{\infty}(X)
$$

is an equivalence of cyclotomic spectra.

Proof. We first observe that the colimits defining the homotopy groups $\pi_{*}^{C_{r}}$ in Section 2.1 may be calculated by restricting to representations of the form $V_{r}^{\oplus k}$, where $V_{r}$ denotes the complex regular representation of $C_{r}$. Thus, in order for the map in the proposition to induce an isomorphism on homotopy groups it suffices that the connectivity of the maps

$$
\Omega^{V_{r}^{\oplus k}}\left(S^{V_{r}^{\oplus k}} \wedge X\right)^{C_{r}} \rightarrow \Omega^{V_{r}^{\oplus k}}\left(\mathbb{E}^{\infty}\left(S^{V_{r}^{\oplus k}} \wedge X\right)\right)^{C_{r}}
$$

tends to infinity with $k$. This was shown to be the case in $[27,7.1]$.

4.3. The combinatorial restriction map. Recall from $[14,7.1]$ that the cyclic bar construction $B_{\bullet}^{\mathrm{cy}}(\Pi)$ associated to a based monoid $\Pi$ is the based cyclic set $[k] \mapsto \Pi^{\wedge(k+1)}$ with simplicial operators

$$
\begin{aligned}
& d_{i}\left(x_{0}, \ldots, x_{k}\right)= \begin{cases}\left(x_{0}, \ldots, x_{i} x_{i+1}, \ldots, x_{k}\right), & 0 \leq i \leq k-1 \\
\left(x_{k} x_{0}, x_{1}, \ldots, x_{k-1}\right), & i=k,\end{cases} \\
& s_{i}\left(x_{0}, \ldots, x_{k}\right)=\left(x_{0}, \ldots, x_{i}, 1_{\Pi}, x_{i+1}, \ldots, x_{k}\right), \quad 0 \leq i \leq k,
\end{aligned}
$$

and cyclic operators $t_{k}\left(x_{0}, \ldots, x_{k}\right)=\left(x_{k}, x_{0}, \ldots, x_{k-1}\right)$. As in the unbased case $[8,2]$ there are canonical isomorphisms of based cyclic sets

$$
R_{r}: \operatorname{sd}_{r} B_{\bullet}^{c y}(\Pi)^{C_{r}} \rightarrow B_{\bullet}^{\mathrm{cy}}(\Pi),
$$

giving $B_{\bullet}^{\mathrm{cy}}(\Pi)$ the structure of a based epicyclic set. Its realization therefore inherits the structure of a cyclotomic space and we have the associated cyclotomic spectrum $\mathbb{E} \Sigma_{\mathbb{T}}^{\infty}\left(B^{\text {cy }}(\Pi)\right)$. Notice that the zeroth space of this spectrum may be identified with the topological realization of the epicyclic set $[k] \mapsto \mathbb{E}_{k}^{\infty}\left(B_{k}^{\text {cy }}(\Pi)\right)$ with structure maps inherited from those of $\mathbb{E}_{\bullet}^{\infty}(-)$ and $B_{\bullet}^{\text {cy }}(\Pi)$. In order to define the combinatorial restriction map we first define a map of epicyclic sets

$$
B_{k}^{\mathrm{cy}}(\Upsilon) \rightarrow B_{k}^{\mathrm{cy}}\left(M_{n}(\Pi)\right) \stackrel{\operatorname{tr}_{k}}{\rightarrow} \mathbb{E}_{k}^{\infty}\left(B_{k}^{\mathrm{cy}}(\Pi)\right)
$$

in which $M_{n}(\Pi)$ denotes the based monoid $\operatorname{Map}\left(\mathbf{n}_{+}, \Pi \wedge \mathbf{n}_{+}\right)$whose multiplication resembles multiplication of $n \times n$-matrices with at most one non-zero element in each column. Let $\phi_{i} \in \Upsilon$ be the element corresponding to the unit of the $i$ th wedge component under the specified isomorphism $\phi: \Pi^{\vee n} \stackrel{\sim}{\rightarrow} \Upsilon$. Then $\vee_{i} \phi_{i} \Pi \cong \Upsilon$ and we may identify $M_{n}(\Pi)$ with the based monoid of based right $\Pi$-maps $\operatorname{Map}_{\Pi}(\Upsilon, \Upsilon)$. Letting $\Upsilon$ act on itself from the left, we get a homomorphism of based monoids

$$
\Upsilon \rightarrow \operatorname{Map}_{\Pi}(\Upsilon, \Upsilon) \cong M_{n}(\Pi), \quad x \mapsto\left(\bar{x}_{i, j}\right) .
$$

Here $\bar{x}_{i, j}$ is the element in $\Pi$ determined by $x \cdot \phi_{j}=\phi_{i} \cdot f\left(\bar{x}_{i, j}\right)$ if $x \cdot \phi_{j}$ is contained in $\phi_{i} \cdot \Pi$ and it is the base point otherwise. The first map in (4.7) is the induced map of cyclic bar constructions. The second map $\operatorname{tr}_{k}$ was first 
introduced in the non-equivariant context of [26]. We shall later need the explicit definition which we now recall. Given a $k$-simplex in $B_{k}^{\text {cy }}\left(M_{n}(\Pi)\right)$ of the form $\left(\left(x_{i, j}^{0}\right), \ldots,\left(x_{i, j}^{k}\right)\right)$, let $D$ be the following set of multi-indices,

$$
D=\left\{\left(s_{0}, \ldots, s_{k}\right): x_{s_{k}, s_{0}}^{0} \neq *, \ldots, x_{s_{k-1}, s_{k}}^{k} \neq *\right\} .
$$

Since by definition the matrices in question have at most one non-base point entry in each column, the projections $\left(s_{0}, \ldots, s_{k}\right) \mapsto s_{i}$ restrict to injective maps $p_{i}: D \rightarrow \mathbf{n}$. Suppose that $D$ has cardinality $m$ and order its elements by choosing a bijection $\gamma: \mathbf{m} \rightarrow D$. The compositions $p_{i} \gamma: \mathbf{m} \rightarrow \mathbf{n}$ are then injective and admit unique factorizations of the form $p_{i} \gamma=\alpha_{i} \sigma_{i}$, where $\alpha_{i}$ is injective and order preserving and $\sigma_{i} \in \Sigma_{m}$. Consider the map $D \rightarrow \Pi^{\wedge(k+1)}$ defined by

$$
\left(s_{0}, \ldots, s_{k}\right) \mapsto\left(x_{s_{k}, s_{0}}^{0}, \ldots, x_{s_{k-1}, s_{k}}^{k}\right)
$$

and let $\mathbf{x}: \mathbf{m} \rightarrow \Pi^{\wedge(k+1)}$ be the composition with $\gamma$. The element

$$
\left(\left(\sigma_{0}, \ldots, \sigma_{k}\right) ; \mathbf{x}\right) \in E_{k} \Sigma_{m} \times B_{k}^{\mathrm{cy}}(\Pi)^{m}
$$

is independent of the choice of $\gamma$ up to multiplication by an element in $\Sigma_{m}$ and the value of $\operatorname{tr}_{k}$ is defined by

$$
\operatorname{tr}_{k}\left(\left(x_{i, j}^{0}\right), \ldots,\left(x_{i, j}^{k}\right)\right)=\left[\left(\sigma_{0}, \ldots, \sigma_{k}\right) ; \mathbf{x}\right] \in \mathbb{E}_{k}^{\infty}\left(B_{k}^{\mathrm{cy}}(\Pi)\right) .
$$

It is not difficult to check that this defines a map of epicyclic sets and topological realization thus gives a map of cyclotomic spaces

$$
B^{\mathrm{cy}}(\Upsilon) \rightarrow \mathbb{E}^{\infty}\left(B^{\mathrm{cy}}(\Pi)\right) .
$$

In order to extend this to a map of cyclotomic spectra we shall use the monad (or triple) multiplication

$$
\mu: \mathbb{E}^{\infty} \mathbb{E}^{\infty}(X) \rightarrow \mathbb{E}^{\infty}(X) .
$$

In $[4,3]$ this is defined to be a natural transformation of endofunctors on the category of based spaces, but the same definition applies to give a natural transformation of the endofunctors on the categories of $\mathbb{T}$-spaces and cyclotomic spaces defined here.

Definition 4.9. The combinatorial restriction map is the map of cyclotomic spectra

$$
f^{*}: \mathbb{E} \Sigma_{\mathbb{T}}^{\infty}\left(B^{\mathrm{cy}}(\Upsilon)\right) \rightarrow \mathbb{E} \Sigma_{\mathbb{T}}^{\infty}\left(B^{\mathrm{cy}}(\Pi)\right)
$$

whose $V$ th component is the composition

$$
\begin{aligned}
\mathbb{E}^{\infty}\left(S^{V} \wedge B^{\mathrm{cy}}(\Upsilon)\right) & \rightarrow \mathbb{E}^{\infty}\left(S^{V} \wedge \mathbb{E}^{\infty}\left(B^{\mathrm{cy}}(\Pi)\right)\right) \\
& \rightarrow \mathbb{E}^{\infty} \mathbb{E}^{\infty}\left(S^{V} \wedge B^{\mathrm{cy}}(\Pi)\right) \stackrel{\mu}{\rightarrow} \mathbb{E}^{\infty}\left(S^{V} \wedge B^{\mathrm{cy}}(\Pi)\right) .
\end{aligned}
$$

Proof of Proposition 4.3. Let $\mathrm{THE}^{\infty}\left(B^{\mathrm{cy}}(\Pi)\right)$ be the cyclotomic spectrum whose $V$ th space is the realization of the cyclic space

$$
[k] \mapsto \underset{\mathcal{I}^{k+1}}{\operatorname{hocolim} \operatorname{Map}}\left(S^{n_{0}} \wedge \cdots \wedge S^{n_{k}}, \mathbb{E}_{k}^{\infty}\left(S^{V} \wedge B_{k}^{\mathrm{cy}}(\Pi) \wedge S^{n_{0}} \wedge \cdots \wedge S^{n_{k}}\right)\right) \text {. }
$$


The construction parallels that of $\mathrm{TH}(\mathbb{S}(\Pi))$ except that the cyclotomic structure maps also involve the cyclotomic structure of $\mathbb{E}^{\infty}$. We apply the analogous definition with $\Upsilon$ instead of $\Pi$ and claim that there is a commutative diagram of cyclotomic spectra

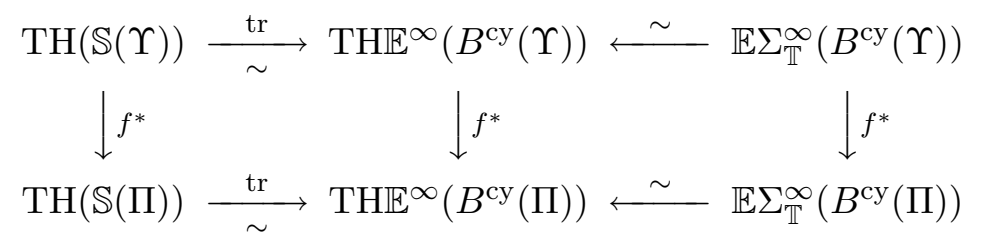

in which the horizontal maps are equivalences. This clearly suffices to prove the proposition. The horizontal maps on the right are the natural inclusion, whereas the definition of the maps tr is analogous to the definition of $\operatorname{tr}_{\bullet}$ in (4.7). In the case of $\Pi$ we first define a map of multi-symmetric spectra

$$
\operatorname{tr}_{k}: V_{k}[\mathcal{F}(\mathbb{S}(\Pi))]\left\langle n_{0}, \ldots, n_{k}\right\rangle \rightarrow \mathbb{E}_{k}^{\infty}\left(S^{V} \wedge B_{k}^{\mathrm{cy}}(\Pi) \wedge S^{n_{0}} \wedge \cdots \wedge S^{n_{k}}\right)
$$

for each $k \geq 0$. Using matrix notation, the domain is a wedge of summands of the form

$$
M_{m_{k}, m_{0}}\left(S^{n_{0}} \wedge \Pi\right) \wedge \cdots \wedge M_{m_{k-1}, m_{k}}\left(S^{n_{k}} \wedge \Pi\right) .
$$

Given an element of the form $\left(\left(x_{i, j}^{0}\right), \ldots,\left(x_{i, j}^{k}\right)\right)$, we define the set of multiindices $D$ and the bijection $\gamma: \mathbf{m} \rightarrow D$ as before. These data specify a $(k+1)$-tuple of permutations $\sigma_{i} \in \Sigma_{m}$ and a map

$$
\mathbf{x}: \mathbf{m} \stackrel{\gamma}{\rightarrow} D \rightarrow S^{n_{0}} \wedge \Pi \wedge \cdots \wedge S^{n_{k}} \wedge \Pi \stackrel{\sim}{\rightarrow} S^{n_{0}} \wedge \cdots \wedge S^{n_{k}} \wedge B_{k}^{\text {cy }}(\Pi) .
$$

The value of $\operatorname{tr}_{k}$ is then defined to be $\left[\left(\sigma_{0}, \ldots, \sigma_{k}\right) ;, \mathbf{x}\right]$. For each representation $V$ we form the smash product with $S^{V}$, use the spectrum structure of $\mathbb{E} \Sigma_{\mathbb{T}}^{\infty}$ and pass to the homotopy colimit over $\mathcal{I}^{k+1}$ to get a cyclic map of the spaces defining the spectra in question. The map tr of cyclotomic spectra is then the induced map of the topological realizations. We apply the analogous definition with $\Upsilon$ instead of $\Pi$ and it remains to prove that the horizontal maps are equivalences. Consider first the chain of maps in the lower part of the diagram. It suffices to show that the natural map from the cyclotomic suspension spectrum $\Sigma_{\mathbb{T}}^{\infty}\left(B^{\text {cy }}(\Pi)\right)$ to each of these spectra is an equivalence. In the case of $\mathbb{E} \Sigma_{\mathbb{T}}^{\infty}\left(B^{\mathrm{cy}}(\Pi)\right)$ this follows from Proposition 4.6 and using the method in the proof of $[8,3.7]$, a similar argument can be applied for $\mathrm{THE}^{\infty}\left(B^{\mathrm{cy}}(\Pi)\right)$. In the case of $\mathrm{TH}(\mathbb{S}(\Pi))$ we first use the non-linear version of $[11,2.1 .5]$ to reduce to Bökstedt's original definition of topological Hochschild homology and we then argue as before. In the same way we see that $\Sigma_{\mathbb{T}}^{\infty}\left(B^{\text {cy }}(\Upsilon)\right)$ is equivalent to the spectra in the upper chain of maps and the conclusion follows. 


\section{CyClic COVERING PROJECtions AND tRAnsfer maps}

By an cyclic covering projection we understand a map of cyclic sets $p_{\bullet}: X_{\bullet} \rightarrow A_{\bullet}$ such that the underlying simplicial map is a simplicial covering projection.

Example 5.1. Let $E_{\bullet}$ be a cyclic set equipped with a free action of a discrete group $G$ and let $H \subseteq G$ be a subgroup of index $n$. Then the projection

$$
p_{\bullet}: E_{\bullet} / H \rightarrow E_{\bullet} / G
$$

is a cyclic covering projection.

Given a cyclic covering projection as above, the topological realization $p:\left|X_{\bullet}\right| \rightarrow\left|A_{\bullet}\right|$ is a $\mathbb{T}$-equivariant covering in the sense that the underlying map of spaces is a topological covering. Restricting the action we get $C_{r^{-}}$ equivariant covering projections for all $r$ and in case we start with a finite $n$-sheeted cyclic covering projection there are associated stable transfer maps

$$
\operatorname{Trf}_{C_{r}}: \Sigma_{C_{r}}\left(\left|A_{\bullet}\right|_{+}\right) \rightarrow \Sigma_{C_{r}}^{\infty}\left(\left|X_{\bullet}\right|_{+}\right)
$$

in the $C_{r}$-equivariant stable homotopy category. We shall define a combinatorial transfer in the form of a map of cyclic $\mathbb{T}$-spectra

$$
\operatorname{Trf} \bullet: \mathbb{E}_{\bullet} \Sigma_{\mathbb{T}}^{\infty}\left(A_{\bullet+}\right) \rightarrow \mathbb{E}_{\bullet} \Sigma_{\mathbb{T}}^{\infty}\left(X_{\bullet+}\right) .
$$

In general, given a cyclic set (or space) $X_{\bullet}$, we let $\mathbb{E}_{\bullet} \Sigma_{\mathbb{T}}^{\infty}\left(X_{\bullet}\right)$ be the cyclic $\mathbb{T}$-spectrum defined by

$$
([k], V) \mapsto \mathbb{E}_{k}^{\infty}\left(S^{V} \wedge X_{k}\right)
$$

with the obvious diagonal cyclic structure. Its topological realization may be identified with $\mathbb{E} \Sigma_{\mathbb{T}}^{\infty}\left(\left|X_{\bullet}\right|\right)$ when we give the latter the usual diagonal $\mathbb{T}$-action. The definition of the combinatorial transfer is dependent on a choice of ordering of each of the fibers of the map $p_{0}: X_{0} \rightarrow A_{0}$ in degree zero. However, we shall prove that it is well-defined up to $C_{r}$-equivariant homotopy for all $r$. Let us write $\mathbb{E} \Sigma_{C_{r}}^{\infty}(-)$ for the $C_{r}$-spectrum obtained by restricting the action.

Proposition 5.2. The topological realization of the combinatorial transfer associated to a finite $n$-sheeted cyclic covering projection $p_{\bullet}: X_{\bullet} \rightarrow A_{\bullet}$ is related to the stable $C_{r}$-equivariant transfer by the following commutative diagram in the $C_{r}$-equivariant stable homotopy category

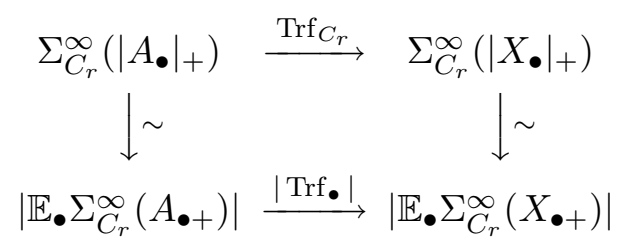

for each finite cyclic group $C_{r}$.

We present the construction of the cyclic transfer in Section 5.1 and establish the compatibility with the equivariant stable transfer in Section 5.2. 
5.1. The combinatorial transfer. The definition of the combinatorial transfer is based on a certain universal property of the cyclic sets $E_{\bullet} \Sigma_{n}$ which we now explain. Let more generally $G$ be any discrete group and let in the following $G$-set mean right $G$-set. Then we may consider the functor $Z \rightarrow E_{\bullet} Z$ from Section 4.2 as a functor from $G$-sets to cyclic $G$-sets by letting $G$ act coordinate-wise. The next lemma expresses the fact that given a cyclic $G$-set $Y_{\bullet}$ and a $G$-set $X$, specifying a map of cyclic $G$-sets $Y_{\bullet} \rightarrow E_{\bullet} X$ is equivalent to specifying a map of $G$-sets $Y_{0} \rightarrow X$.

Lemma 5.3. The functor $E_{\bullet}$ from $G$-sets to cyclic $G$-sets is right adjoint to the functor that takes a cyclic $G$-sets to its 0 th space.

Proof. We begin by making some remarks on the vertices of a $k$-simplex $y$ in a cyclic set $Y_{\bullet}$. By definition, these are the 0 -simplices $\bar{y}(0), \ldots, \bar{y}(k)$, where $\bar{y}: \Delta_{\bullet}[k] \rightarrow Y_{\bullet}$ is the characteristic map determined by $y$ and $(i):[0] \rightarrow[k]$ maps 0 to $i$. It follows from the defining relations for a cyclic set that the cyclic operator $t_{k}$ on $Y_{k}$ acts by cyclic permutation on the vertices in the sense that

$$
\left(\overline{t_{k} y}(0), \ldots, \overline{t_{k} y}(k)\right)=(\bar{y}(k), \bar{y}(0), \ldots, \bar{y}(k-1)) .
$$

Suppose now that $f_{0}: Y_{0} \rightarrow X$ is a map of $G$-sets. This has a unique extension to a map of simplicial $G$-sets $f_{\bullet}: Y_{\bullet} \rightarrow E_{\bullet} X$ given by

$$
f_{k}(y)=\left(f_{0}(\bar{y}(0)), \ldots, f_{0}(\bar{y}(k))\right)
$$

By the above remark this is in fact a map of cyclic sets as required.

Let now $p_{\bullet}: X_{\bullet} \rightarrow A_{\bullet}$ be an $n$-sheeted covering projection of cyclic sets and let $P\left(X_{\bullet}\right) \rightarrow A_{\bullet}$ be the associated cyclic principal $\Sigma_{n}$-bundle. By definition,

$$
P\left(X_{k}\right)=\left\{\left(x_{1}, \ldots, x_{n}\right) \in X_{k}^{n}: p_{k}\left(x_{i}\right)=p_{k}\left(x_{j}\right) \text { and } x_{i} \neq x_{j} \text { for } i \neq j\right\}
$$

and the cyclic structure of $P\left(X_{\bullet}\right)$ is induced from that of the diagonal cyclic set $X_{\bullet}^{n}$. The symmetric group $\Sigma_{n}$ acts freely by permutation of the coordinates and $P\left(X_{\bullet}\right) / \Sigma_{n}$ may be identified with $A_{\bullet}$. It follows from Lemma 5.3 that specifying a $\Sigma_{n}$-equivariant map of cyclic sets $P\left(X_{\bullet}\right) \rightarrow E_{\bullet} \Sigma_{n}$ is equivalent to specifying a $\Sigma_{n}$-equivariant map of sets $P\left(X_{0}\right) \rightarrow \Sigma_{n}$. This in turn is equivalent to choosing an ordering of each of the fibers of the map $p_{0}: X_{0} \rightarrow A_{0}$. Suppose that such choices have been made and consider the $\Sigma_{n}$-equivariant cyclic map

$$
P\left(X_{\bullet}\right) \rightarrow E_{\bullet} \Sigma_{n} \times X_{\bullet}^{n}
$$

in which the target is the diagonal cyclic set and the map to the second factor is the natural inclusion. Passing to $\Sigma_{n}$-orbits we obtain a cyclic map

$$
A_{\bullet} \cong P\left(X_{\bullet}\right) / \Sigma_{n} \rightarrow E_{\bullet} \Sigma_{n} \times_{\Sigma_{n}} X_{\bullet}^{n} \rightarrow \mathbb{E}_{\bullet}^{\infty}\left(X_{\bullet+}\right),
$$

where the target again denotes the diagonal cyclic set and the last map is the natural inclusion. In order to extend this to a map of cyclic $\mathbb{T}$-spectra we use the $\mathbb{E}_{\bullet}^{\infty}$ monad multiplication as in Definition 4.9. 
Definition 5.4. The combinatorial transfer determined by the chosen ordering of the fibers of $p_{0}$ is the map of cyclic $\mathbb{T}$-spectra

$$
\operatorname{Trf}_{\bullet}: \mathbb{E}_{\bullet} \Sigma_{\mathbb{T}}^{\infty}\left(A_{\bullet+}\right) \rightarrow \mathbb{E}_{\bullet} \Sigma_{\mathbb{T}}^{\infty}\left(X_{\bullet+}\right)
$$

whose $V$ th component in simplicial degree $k$ is the composition

$$
\begin{aligned}
\mathbb{E}_{k}^{\infty}\left(S^{V} \wedge A_{k+}\right) & \rightarrow \mathbb{E}_{k}^{\infty}\left(S^{V} \wedge \mathbb{E}_{k}^{\infty}\left(X_{k+}\right)\right) \\
& \rightarrow \mathbb{E}_{k}^{\infty} \mathbb{E}_{k}^{\infty}\left(S^{V} \wedge X_{k+}\right) \stackrel{\mu}{\rightarrow} \mathbb{E}_{k}^{\infty}\left(S^{V} \wedge X_{k+}\right) .
\end{aligned}
$$

Lemma 5.5. The topological realization of the combinatorial transfer is independent of the choice of ordering up to $C_{r}$-equivariant homotopy for all $r \geq 1$.

Proof. Using lemma 5.3 it is easy to see that the cyclic maps $A_{\bullet} \rightarrow \mathbb{E}_{\bullet}^{\infty}\left(X_{\bullet}\right)$ associated to different orderings are related by special homotopies in the sense of Section 4.1. The result then follows from the general discussion in that section.

It should be remarked that in general one cannot find a $\mathbb{T}$-equivariant homotopy in the above lemma.

5.2. Compatibility with the equivariant stable transfer. We begin by recalling the construction of the equivariant stable transfer associated to an $n$-sheeted $G$-equivariant covering projection $p: X \rightarrow A$ in the case where $G$ is a finite group and $A$ and $X$ are $G$-CW complexes. Let $V_{G}$ be the real regular representation of $G$ equipped with the usual inner product. We shall generalize the non-equivariant approach in $[2,1.4]$ to the equivariant setting by using the unit disc $D_{G}=D\left(V_{G}\right)$ as a substitute for the unit interval throughout. Suppose first that $A$ and $X$ are finite dimensional. Then there exists a $G$-equivariant map $\varphi: X \rightarrow V_{G}^{n}$ such that (i) the image $\varphi(X)$ is contained in the interior of the product $D_{G}^{n}$ and (ii) the map

$$
(p, \varphi): X \rightarrow A \times D_{G}^{n}
$$

is a $G$-equivariant embedding. We extend this to a $G$-equivariant embedding

$$
X \times D_{G}^{n} \rightarrow A \times D_{G}^{n}, \quad(x, v) \mapsto(p(x), e(x, v)),
$$

such that for each fixed $x$ the second factor $e(x)$ is a product of little discs in $D_{G}$ with centers $\varphi_{i}(x)$, that is, a product of affine maps of the form

$$
e(x): D_{G} \rightarrow D_{G}, \quad e(x, v)=\varphi_{i}(x)+r_{i}(x) v,
$$

where $0<r_{i}(x)$. Identifying $D_{G} / \partial D_{G}$ with the one-point compactification $S^{V_{G}}$, the usual Pontryagin-Thom construction gives a $G$-equivariant map in the opposite direction

$$
S^{V_{G}^{n}} \wedge A_{+} \rightarrow S^{V_{G}^{n}} \wedge X_{+}
$$

The adjoint of this is a $G$-map $A_{+} \rightarrow \Omega^{V_{G}^{n}}\left(S^{V_{G}^{n}} \wedge X_{+}\right)$, which is independent of choices up to equivariant homotopy. In the case where $A$ and $X$ are not 
finite dimensional we make compatible choices for each skeleton and get a map

$$
A_{+} \rightarrow Q_{G}\left(X_{+}\right)=\operatorname{colim}_{n} \Omega^{V_{G}^{n}}\left(S_{G}^{V_{G}^{n}} \wedge X_{+}\right) .
$$

The equivariant stable transfer is the corresponding map of spectra

$$
\operatorname{Trf}_{C_{r}}: \Sigma_{C_{r}}^{\infty}\left(A_{+}\right) \rightarrow \Sigma_{C_{r}}^{\infty}\left(X_{+}\right)
$$

in the $C_{r}$-equivariant stable homotopy category. In order to compare this construction to that of the cyclic transfer we reformulate the above in terms of the $G$-equivariant little product discs operad $\mathcal{C}_{G}^{\infty}$. As above we define a little product disc $\omega$ in $D_{G}^{n}$ to be a product of affine maps of the form

$$
\omega_{i}: D_{G} \rightarrow D_{G}, \quad \omega_{i}(v)=v_{i}+r_{i} v,
$$

where $v_{i} \in D_{G}$ and $0<r_{i}$. Let $\mathcal{C}_{G}^{n}(k)$ be the space of $k$-tuples of pairwise disjoint little product discs in $D_{G}^{n}$ and let

$$
\mathcal{C}_{G}^{\infty}(k)=\underset{n}{\operatorname{colim}} \mathcal{C}_{G}^{n}(k) .
$$

This is an equivariant analogue of the little cubes operad [23] and the spaces $\mathcal{C}_{G}^{\infty}(k)$ form a $G$-equivariant operad in the sense of [16, VII] with $\Sigma_{k}$ acting by permuting the little product $\operatorname{discs}$ and $G$ acting by conjugation. It follows from $[13,5.3]$ that this is an $E_{\infty} G$-operad in the sense that (i) the $\Sigma_{k}$-action on $\mathcal{C}_{G}^{\infty}(k)$ is free for each $k$ and (ii) the fixed-point spaces $\mathcal{C}_{G}^{\infty}(k)^{H}$ are contractible for subgroups $H \subseteq G \times \Sigma_{n}$ satisfying that $H \cap \Sigma_{n}=\left\{1_{H}\right\}$. These are exactly the conditions for the spaces $\mathcal{C}_{G}^{\infty}(k)$ to be universal $\left(G, \Sigma_{k}\right)$-principal bundles. Given a $G$-equivariant $n$-sheeted covering projection $p: X \rightarrow A$ as above, the associated $\left(G, \Sigma_{n}\right)$-principal bundle $P(X)$ is thus classified by a $G \times \Sigma_{n}$-equivariant map $P(X) \rightarrow \mathcal{C}_{G}^{\infty}(n)$, uniquely determined up to equivariant homotopy. It is clear from the definitions that the transfer as defined in (5.6) may be identified with the composition

$$
A \cong P(X) / \Sigma_{n} \rightarrow \mathcal{C}_{G}^{\infty}(n) \times_{\Sigma_{n}} X^{n} \rightarrow \mathcal{C}_{G}^{\infty}(n) \times_{\Sigma_{n}} Q_{G}\left(X_{+}\right)^{n} \rightarrow Q_{G}\left(X_{+}\right)
$$

in which the first map is analogous to that used in the definition of the combinatorial transfer and the last map is induced by the natural action of $\mathcal{C}_{G}^{\infty}$ on $Q_{G}\left(X_{+}\right)$, cf. $[23,5.1]$. Let us write $\mathcal{E}_{C_{r}}^{\infty}$ for the $C_{r}$-equivariant version of the Barratt-Eccles operad [23,6.4] defined by letting $\mathcal{E}_{C_{r}}^{\infty}(k)=E \Sigma_{k}$ with the $C_{r}$-action induced by the cyclic structure.

Lemma 5.7. The operad $\mathcal{E}_{C_{r}}^{\infty}$ is an $E_{\infty} C_{r}$-operad.

Proof. This follows from the proof of Theorem 1.3 given in Section 6.1 by letting $x=1_{\Sigma_{k}}$.

Proof of Proposition 5.2. Let $p: X \rightarrow A$ be the $C_{r}$-equivariant $n$-sheeted topological covering projection obtained by topological realization of the cyclic covering projection in question. Recall from [16, VII] that any $C_{r^{-}}$ equivariant operad $\mathcal{C}$ defines a monad $C$ on the category of based $C_{r}$-spaces 
by letting

$$
C(Z)=\coprod_{k=0}^{\infty} \mathcal{C}(k) \times_{\Sigma_{k}} Z^{k} / \sim .
$$

By definition, $\mathbb{E}_{C_{r}}^{\infty}$ is the monad associated to the operad $\mathcal{E}_{C_{r}}^{\infty}$ and the above equivalence relation is defined analogously in general. Letting $C_{C_{r}}^{\infty}$ be the monad associated to the little product discs operad $\mathcal{C}_{C_{r}}^{\infty}$, we may write the transfer (5.6) as the composition

$$
A \cong P(X) / \Sigma_{n} \rightarrow \mathcal{C}_{C_{r}}^{\infty}(n) \times_{\Sigma_{n}} X^{n} \rightarrow C_{C_{r}}^{\infty}\left(X_{+}\right) \rightarrow Q_{C_{r}}\left(X_{+}\right),
$$

where the last map is induced by the action of $\mathcal{C}_{C_{r}}^{\infty}$ on $Q_{C_{r}}\left(X_{+}\right)$. Let now $\mathcal{D}_{C_{r}}^{\infty}$ be the $C_{r^{-}}$equivariant product operad $\mathcal{C}_{C_{r}}^{\infty} \times \mathcal{E}_{C_{r}}^{\infty}$ and let $D_{C_{r}}^{\infty}$ be the associated monad. By Lemma 5.7 the projections

$$
\mathcal{C}_{C_{r}}^{\infty} \leftarrow \mathcal{D}_{C_{r}}^{\infty} \rightarrow \mathcal{E}_{C_{r}}^{\infty}
$$

are maps of $E_{\infty} C_{r}$-operads and by the equivariant version of [24, A.2] it follows that the induced maps of monads are $C_{r}$-equivariant equivalences. Consider now the commutative diagram

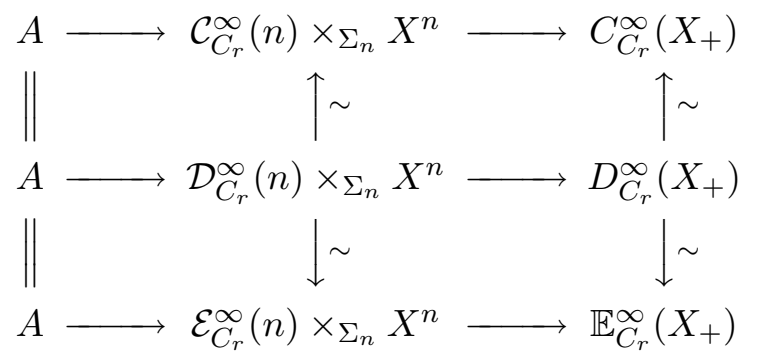

in which the vertical maps are $C_{r}$-equivariant equivalences by the above remarks. The result now follows by extending this to a diagram of spectra and passing to the $C_{r}$-equivariant stable homotopy category.

\section{GRoup RINGS AND RESTRICTION MAPS}

In this section we prove the theorems from Section 1 with the exception of Theorem 1.6, which is proved in Section 7. Given a discrete group $G$, let $B_{\bullet}^{\text {cy }}(G)$ be the unbased version of the bar construction $[8,2]$ and let $B^{\text {cy }}(G)$ be the topological realization. The based version $B^{\mathrm{cy}}\left(G_{+}\right)$considered in Section 4.3 may be identified with $B^{\mathrm{cy}}(G)_{+}$. Suppose that $H \subseteq G$ is a subgroup of finite index, say $|G / H|=n$. Specifying an isomorphism of right $H$-sets $\phi: \sqcup_{i=1}^{n} H \rightarrow G$ that restricts to the inclusion on the first component then amounts to choosing a transversal of $H$ in $G$, ie, a choice of left coset representatives $\phi_{i} \in G$ such that $\phi_{1}$ is the unit of $H$. We shall analyze the combinatorial restriction map

$$
\operatorname{Res}_{G}^{H}: \mathbb{E} \Sigma_{\mathbb{T}}^{\infty}\left(B^{\mathrm{cy}}(G)_{+}\right) \rightarrow \mathbb{E} \Sigma_{\mathbb{T}}^{\infty}\left(B^{\mathrm{cy}}(H)_{+}\right)
$$

from Section 4.3 in terms of the combinatorial transfer maps introduced in Section 5.1. Given a conjugacy class $\omega$ of elements in $G$, let $B_{\bullet}^{\text {cy }}(G, \omega)$ be 
the cyclic subset defined by

$$
B_{k}^{\mathrm{cy}}(G, \omega)=\left\{\left(g_{0}, \ldots, g_{k}\right): g_{0} \cdots g_{k} \in \omega\right\}
$$

and let $B^{\mathrm{cy}}(G, \omega)$ be its topological realization. Then $B_{\bullet}^{\mathrm{cy}}(G)$ splits as the disjoint union

$$
B_{\bullet}^{\mathrm{cy}}(G)=\coprod_{\omega \in\langle G\rangle} B_{\bullet}^{\mathrm{cy}}(G, \omega)
$$

indexed on the set of conjugacy classes $\langle G\rangle$ and $B^{\text {cy }}(G)$ splits accordingly.

Proposition 6.2. The splitting in (6.1) gives rise to an equivalence of cyclotomic spectra

$$
\mathbb{E} \Sigma_{\mathbb{T}}^{\infty}\left(B^{c y}(G)_{+}\right) \stackrel{\sim}{\rightarrow} \prod_{\omega \in\langle G\rangle}^{\prime} \mathbb{E} \Sigma_{\mathbb{T}}^{\infty}\left(B^{c y}(G, \omega)_{+}\right),
$$

where $\Pi^{\prime}$ denotes the weak product.

Proof. We must show that the target admits the structure of a cyclotomic spectrum compatible with that of the domain. Notice first that the cyclotomic structure maps of $B^{\mathrm{cy}}(G)$ restrict to homeomorphism

$$
\rho_{r}^{*} B^{\mathrm{cy}}(G, \omega)^{C_{r}} \rightarrow \coprod_{\left\{v \in\langle G\rangle: v^{r}=\omega\right\}} B^{\mathrm{cy}}(G, v) .
$$

The cyclotomic structure maps on the target are then products over $\omega \in\langle G\rangle$ of maps of the form

$$
\begin{aligned}
& R_{r}^{\omega}(V): \rho_{r}^{*} \mathbb{E}^{\infty}\left(S^{V} \wedge B^{\mathrm{cy}}(G, \omega)_{+}\right)^{C_{r}} \rightarrow \mathbb{E}^{\infty}\left(S^{\rho_{r}^{*} V^{C_{r}}} \wedge \rho_{r}^{*} B^{\mathrm{cy}}(G, \omega)_{+}^{C_{r}}\right) \\
\rightarrow & \mathbb{E}^{\infty}\left(\bigvee_{\left\{v: v^{r}=\omega\right\}} S^{\rho_{r}^{*} V^{C_{r}}} \wedge B^{\mathrm{cy}}(G, v)_{+}\right) \rightarrow \prod_{\left\{v: v^{r}=\omega\right\}} \mathbb{E}^{\infty}\left(S^{\rho_{r}^{*} V^{C_{r}}} \wedge B^{\mathrm{cy}}(G, v)_{+}\right),
\end{aligned}
$$

where the second map is induced by the above homeomorphism.

We have an analogous splitting of $\mathbb{E} \Sigma_{\mathbb{T}}^{\infty}\left(B^{\text {cy }}(H)_{+}\right)$and define

$$
\operatorname{Res}_{G}^{H}(\lambda, \omega): \mathbb{E} \Sigma_{\mathbb{T}}^{\infty}\left(B^{\mathrm{cy}}(G, \omega)_{+}\right) \rightarrow \mathbb{E} \Sigma_{\mathbb{T}}^{\infty}\left(B^{\mathrm{cy}}(H, \lambda)_{+}\right)
$$

as in Section 1.2. Recall the notion of a special homotopy from Section 4.1. In the next section we show that if $\lambda \subseteq \omega$, then the inclusion of the cyclic sets $B_{\bullet}^{\text {cy }}(H, \lambda) \rightarrow B_{\bullet}^{\text {cy }}(G, \omega)$ is equivalent to a cyclic covering projection up to special homotopy equivalence and in Section 6.2 we compare $\operatorname{Res}_{G}^{H}(\lambda, \omega)$ to the associated combinatorial transfer.

6.1. Centralizers and cyclic covering projections. Given a discrete group $G$, let $G^{\text {ad }}$ be the left $G$-set obtained by letting $G$ act on itself by conjugation. There is a simplicial isomorphism

$$
\Phi_{\bullet}: B_{\bullet}^{\mathrm{cy}} G \stackrel{\sim}{\rightarrow} E_{\bullet} G \times{ }_{G} G^{\mathrm{ad}}
$$

defined by

$$
\Phi_{k}\left(g_{0}, \ldots, g_{k}\right)=\left[\left(g\langle 1, k\rangle, g\langle 2, k\rangle \ldots, g\langle k, k\rangle, 1_{G}\right), g\langle 0, k\rangle\right],
$$


where $g\langle i, k\rangle$ denotes the product $g_{i} \cdots g_{k}$ and $1_{G}$ is the unit of $G$. Under this isomorphism the cyclic operators of $B_{\bullet}^{\text {cy }} G$ correspond to the cyclic operators

$$
t_{k}\left[\left(g_{0}, \ldots, g_{k}\right), z\right]=\left[\left(g_{k} z, g_{0}, \ldots, g_{k-1}\right), z\right] .
$$

It is clear that $\Phi$. restricts to give an isomorphism

$$
\Phi_{\bullet}^{\omega}: B_{\bullet}^{\mathrm{cy}}(G, \omega) \stackrel{\sim}{\rightarrow} E_{\bullet} G \times_{G} \omega
$$

for each conjugacy class $\omega$ in $G$. Let now $x \in \omega$ be a representative. Then $x$ specifies an isomorphism of left $G$-sets $G / C_{G}(x) \rightarrow \omega$, where $C_{G}(x)$ denotes the centralizer of $x$ in $G$. Consequently, $\Phi_{\bullet}^{\omega}$ gives rise to a simplicial isomorphism

$$
\Phi_{\bullet}^{x}: B_{\bullet}^{\text {cy }}(G, \omega) \stackrel{\sim}{\rightarrow} E_{\bullet} \times_{G} \omega \stackrel{\sim}{\rightarrow} E_{\bullet} G \times_{G} G / C_{H}(x) \stackrel{\sim}{\rightarrow} E_{\bullet} G / C_{G}(x) .
$$

Choosing an element $z \in G$ such that $z x z^{-1}=g\langle 0, k\rangle$, we get the explicit expression

$$
\Phi_{k}^{x}\left(g_{0}, \ldots, g_{k}\right)=[g\langle 1, k\rangle z, \ldots, g\langle k, k\rangle z, z] .
$$

Under this isomorphism the cyclic operators of $E_{\bullet} G / C_{G}(x)$ act by

$$
t_{k}\left[g_{0}, \ldots, g_{k}\right]=\left[g_{k} x, g_{0}, \ldots, g_{k-1}\right] .
$$

Definition 6.5. Given a element $x \in G$, we let $E_{\bullet}(G, x) / C_{G}(x)$ be the cyclic set $E_{\bullet} G / C_{G}(x)$ equipped with the cyclic operators (6.4).

We may view the topological realization $E(G, x) / C_{G}$ as a model of the classifying space $B C_{G}(x)$ equipped with a certain $\mathbb{T}$-action induced by the cyclic structure. Using the model $E(G, x)$ we give an easy proof of Lydakis' Theorem 1.3 characterizing the $C_{r}$-equivariant homotopy type.

Proof of Theorem 1.3. We apply the edgewise subdivision functor to get a simplicial $C_{r}$-set

$$
\operatorname{sd}_{r}\left(E_{\bullet}(G, x) / C_{G}(x)\right)=E_{\bullet}(G, x)^{r} / C_{G}(x)
$$

in which the generator $\xi$ of $C_{r}$ acts by

$$
\left[e_{1}, \ldots, e_{r}\right] \cdot \xi=\left[e_{r} x, e_{1}, \ldots, e_{r-1}\right] .
$$

Let $E_{\bullet}=E_{\bullet} G^{r}$ and extend the diagonal right $C_{G}(x)$-action to an action of $C_{G}(x)\left\{x^{1 / r}\right\}$ by letting the extra generator $\zeta$ act by

$$
\left(e_{1}, \ldots, e_{r}\right) \cdot \zeta=\left(e_{r} x, e_{1}, \ldots, e_{r-1}\right) .
$$

We claim that the realization $E$ is a universal $C_{G}(x)$-free $C_{G}(x)\left\{x^{1 / r}\right\}$-space, that is, that $E^{K}$ is contractible for subgroups $K \subseteq C_{G}(x)\left\{x^{1 / r}\right\}$ satisfying that $K \cap C_{G}(x)=\left\{1_{K}\right\}$. The composition

$$
K \rightarrow C_{G}(x)\left\{x^{1 / r}\right\} \rightarrow C_{r}
$$

is injective for such $K$, hence $K$ is a cyclic group whose order divides $r$. Let $|K|=n$ and choose a generator of the form $t=z \zeta^{m}$ with $z \in C_{G}(x)$ and $m=r / n$. Writing $E=\left((E G)^{m}\right)^{n}$, the action of $t$ is given by

$$
\left(e_{1}, \ldots, e_{n}\right) \cdot t=\left(e_{n} z x, e_{1} z, \ldots, e_{n-1} z\right), \quad e_{1}, \ldots, e_{n} \in E G^{m},
$$


and the condition for $\left(e_{1}, \ldots, e_{n}\right)$ to be fixed is that

$$
e_{1}=e_{n} z x, e_{2}=e_{n} z^{2} x, \ldots, e_{n}=e_{n} z^{n} x .
$$

Since $z^{n} x=t^{n}=1_{K}$, we find that $E^{K}=E G^{m} \simeq *$.

Example 6.6. For $x=1_{G}$, it follows from the discussion in Section 1.3 that $C_{G}(x)\left\{x^{1 / r}\right\}=G \times C r$. This case of the above proposition was also considered in $[8,7.13]$. Suppose now that $G$ is torsion free. Then the only subgroups $K \subseteq G \times C_{r}$ satisfying that $K \cap G=\left\{1_{K}\right\}$ are the subgroups of $C_{r}$ and it follows that $E G$ equipped with the trivial $C_{r}$-action is a universal $G$-free $G \times C_{r}$-space. Consequently, the inclusion

$$
B^{\mathrm{cy}}\left(G, 1_{G}\right)^{C_{r}} \rightarrow B^{\mathrm{cy}}\left(G, 1_{G}\right)
$$

is a $C_{r}$-equivariant homotopy equivalence.

Suppose now that $H \subseteq G$ is a subgroup of finite index and that $\lambda \in\langle H\rangle$ is a conjugacy class contained in $\omega$. Given a representative $x \in \lambda$, we have an isomorphism analogous to (6.3), and under these isomorphisms the inclusion $B_{\bullet}^{\text {cy }}(H, \lambda) \rightarrow B_{\bullet}^{\text {cy }}(G, \omega)$ corresponds to the natural map

$$
E_{\bullet}(H, x) / C_{H}(x) \rightarrow E_{\bullet}(G, x) / C_{G}(x) .
$$

Lemma 6.7. The inclusion

$$
E_{\bullet}(H, x) / C_{H}(x) \rightarrow E_{\bullet}(G, x) / C_{H}(x)
$$

is a special homotopy equivalence.

Proof. Notice first that the cyclic operators introduced in (6.4) extend to give $E_{\bullet}(G, x) / C_{H}(x)$ the structure of a cyclic set. We shall use the choice of left coset representatives $\phi_{i} \in G$ to define a retraction

$$
r_{\bullet}: E_{\bullet}(G, x) / C_{H}(x) \rightarrow E_{\bullet}(H, x) / C_{H}(x) .
$$

Such representatives determine an isomorphism of right $H$-sets $r_{0}: G \rightarrow H$ and using Lemma 5.3, this has a unique extension to an $H$-equivariant cyclic map

$$
r_{\bullet}: E_{\bullet} G \rightarrow E_{\bullet} H, \quad r_{k}\left(g_{0}, \ldots, g_{k}\right)=\left(r_{0}\left(g_{0}\right), \ldots, r_{0}\left(g_{k}\right)\right) .
$$

The retraction (6.8) is the induced map of orbit spaces. Since $r_{0}$ is $H$ equivariant, it remains a map of cyclic sets when the cyclic operators are twisted by $x$. Applying Lemma 5.3 once more, we get a special homotopy

$$
E_{\bullet}(G, x) / C_{H}(x) \times B_{\bullet}^{\text {cy }}(J) \rightarrow E_{\bullet}(G, x) / C_{H}(x)
$$

relating the identity and the composition of the retraction followed by the inclusion.

It follows from the above lemma that the topological realization of the cyclic map in question is a $C_{r}$-equivariant homotopy equivalence for all $r$. 
Consider now the following diagram of cyclic sets

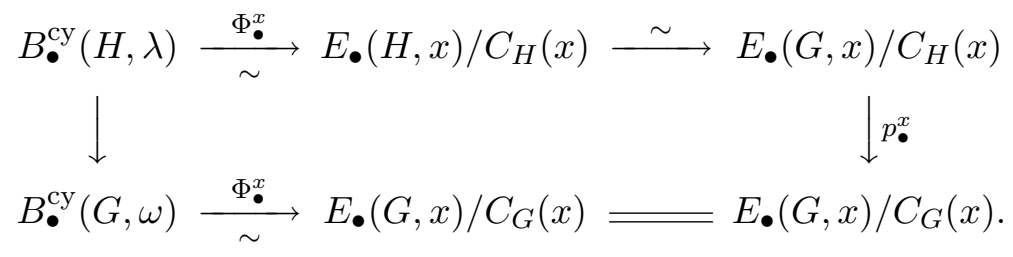

associated to a pair of conjugacy class $\lambda \in\langle H\rangle, \omega \in\langle G\rangle$ and a representative $x \in \lambda$. Using Lemma 6.7 it follows that the inclusion on the left hand side is equivalent to the cyclic covering projection $p_{\bullet}^{x}$ on the right hand side up to special homotopy equivalence.

6.2. The proofs of Theorem 1.1, 1.2 and 1.4. We first compare the combinatorial restriction $\operatorname{map}^{\operatorname{Res}_{G}^{H}}(\lambda, \omega)$ to the realization of the combinatorial transfer

$$
\operatorname{Trf} \bullet: \mathbb{E}_{\bullet} \Sigma_{\mathbb{T}}^{\infty}\left(E_{\bullet}(G, x) / C_{G}(x)_{+}\right) \rightarrow \mathbb{E}_{\bullet} \Sigma_{\mathbb{T}}^{\infty}\left(E_{\bullet}(G, x) / C_{H}(x)_{+}\right)
$$

associated to the cyclic covering projection $p_{\bullet}^{x}$ introduced in Diagram (6.9). Recall that this transfer is dependent on a choice of ordering of each fiber of the projection

$$
p_{0}^{x}: G / C_{H}(x) \rightarrow G / C_{G}(x) .
$$

We claim that the choice of coset representatives $\phi_{i}$ of $G / H$ give canonical choices of such orderings. Indeed, restricting to the fiber over a $\operatorname{coset} g C_{G}(x)$ we get an injective map

$$
\left(p_{0}^{x}\right)^{-1}\left\{g C_{G}(x)\right\} \rightarrow G / C_{H}(x) \rightarrow G / H
$$

and the fiber thus inherits an ordering from the ordered set $G / H$. We define Trf $\bullet$ above to be the cyclic transfer associated to these choices of orderings.

Theorem 6.10. Let there be given a pair of conjugacy classes $\lambda \in\langle H\rangle$ and $\omega \in\langle G\rangle$.

(i): Suppose that $\lambda \nsubseteq \subseteq \omega$. Then $\operatorname{Res}_{G}^{H}(\lambda, \omega)=*$.

(ii): Suppose that $\lambda \subseteq \omega$ and let $x \in \lambda$ be a representative. Then there is a strictly commutative diagram of $\mathbb{T}$-spectra

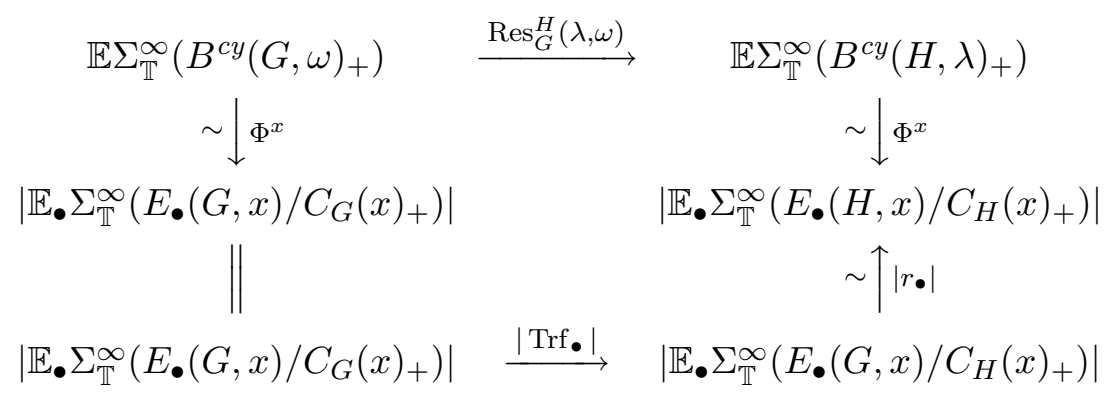

in which the combinatorial transfer $\operatorname{Trf} \bullet$ is defined using the choices specified above and $r_{\bullet}$ is the special homotopy equivalence defined in (6.8). 
Proof. By naturality of the $\mathbb{E}^{\infty}$-monad structure it suffices to consider the underlying maps of cyclic sets in spectrum degree zero. We first introduce some notation convenient for writing down explicit expressions for these maps. A dot - will indicate group multiplication in $G$ and $H$. Given the choice of coset representatives $\phi_{i}$, an element $g \in G$ determines a permutation $i(g) \in \Sigma_{n}$ and elements $\bar{g}(j) \in H$ for $j=1, \ldots, n$ by the requirements that

$$
g \cdot \phi_{j}=\phi_{i(g)(j)} \cdot \bar{g}(j)
$$

Then, in the notation of (4.8), the homomorphism of pointed monoids $G_{+} \rightarrow$ $M_{n}\left(H_{+}\right)$maps an element $g$ to the matrix $\left(\bar{g}_{i, j}\right)$ defined by

$$
\bar{g}_{i, j}= \begin{cases}\bar{g}(j), & \text { for } i=i(g)(j) \\ *, & \text { for } i \neq i(g)(j) .\end{cases}
$$

Let now $\left(g_{0}, \ldots, g_{k}\right)$ be an element of $B_{k}^{\text {cy }}(G, \omega)$ and recall the notation $g\langle i, k\rangle$ for the product $g_{i} \cdots g_{k}$. With the above notation we have the equality

$$
\overline{g\langle i, k\rangle}(j)=\bar{g}_{i}(i(g\langle i+1, k\rangle)(j)) \cdots \bar{g}_{k-1}(i(g\langle k, k\rangle)(j)) \cdot \bar{g}_{k}(j) .
$$

Consider the set

$$
\mathcal{S}=\left\{j \in n: \phi_{j}^{-1} \cdot g\langle 0, k\rangle \cdot \phi_{j} \in \lambda\right\}
$$

and notice that this is non-empty exactly when $\lambda \subseteq \omega$. We denote its cardinality by $m$ and let $\alpha: \mathbf{m} \rightarrow \mathbf{n}$ be the unique order preserving injective map whose image is $\mathcal{S}$. Using the above notation,

$$
\operatorname{Res}_{G}^{H}(\lambda, \omega)\left(g_{0}, \ldots, g_{k}\right) \in \mathbb{E}_{k}^{\infty}\left(B_{k}^{\mathrm{cy}}(H, \lambda)_{+}\right)
$$

is represented by

$$
\left(\left(\alpha_{0}, \ldots, \alpha_{k}\right),\left(\mathbf{x}_{1}, \ldots, \mathbf{x}_{m}\right)\right) \in \Sigma_{m}^{k+1} \times B_{k}^{\mathrm{cy}}(H, \lambda)^{m},
$$

where

$$
\alpha_{i}= \begin{cases}\alpha^{*}(i(g\langle i+1, k\rangle)), & \text { for } 0 \leq i<k \\ 1_{m}, & \text { for } i=k\end{cases}
$$

and

$$
\mathbf{x}_{s}=\left(\bar{g}_{0}(i(g\langle 1, k\rangle))(\alpha(s)), \ldots, \bar{g}_{k-1}(i(g\langle k, k\rangle))(\alpha(s)), \bar{g}_{k}(\alpha(s))\right)
$$

for $s=1, \ldots, m$. If $\lambda \nsubseteq \omega$ this represents the base point and (i) follows. Suppose now that $\lambda \subseteq \omega$ and that $x \in \lambda$ is a representative. We must show 
that there is a commutative diagram of cyclic sets

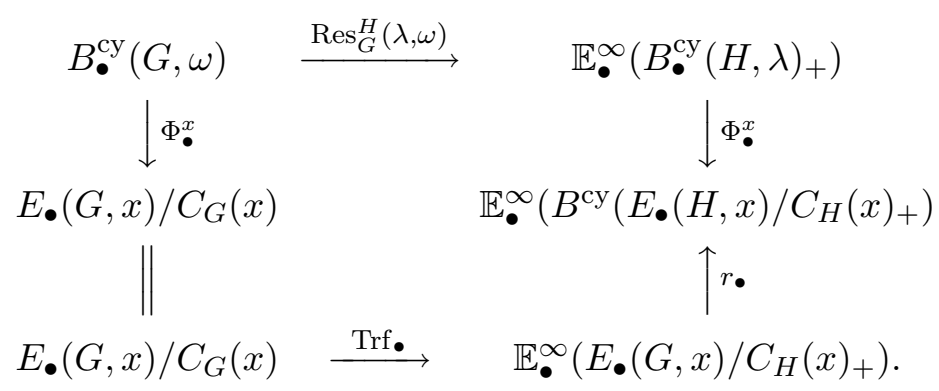

In order to calculate the image of $\Phi_{k}^{x}$ applied to the representative in (6.11) we choose elements $h_{s} \in H$ such that

$$
h_{s} \cdot x \cdot h_{s}^{-1}=\overline{g\langle 0, k\rangle}(\alpha(s))
$$

for $s=1, \ldots, m$. Then, by the definition of $\Phi_{\bullet}^{x}$, its value on (6.11) is represented by

$$
\left(\left(\alpha_{0}, \ldots, \alpha_{k}\right),\left(\mathbf{y}_{1}, \ldots, \mathbf{y}_{m}\right)\right) \in \Sigma_{m}^{k+1} \times\left(E_{k}(G, x) / C_{G}(x)\right)^{m},
$$

where

$$
\mathbf{y}_{s}=\left(\overline{g\langle 1, k\rangle}(\alpha(s)) \cdot h_{s}, \ldots, \overline{g\langle k, k\rangle}(\alpha(s)) \cdot h_{s}, h_{s}\right) .
$$

We now proceed to show that chasing the $k$-simplex $\left(g_{0}, \ldots, g_{k}\right)$ the other way round the diagram gives the same result. We have

$$
\Phi_{k}^{x}\left(g_{0}, \ldots, g_{k}\right)=[g\langle 1, k\rangle \cdot z, \ldots, g\langle k, k\rangle \cdot z, z],
$$

where $z$ is any element in $G$ such that $z \cdot x \cdot z^{-1}=g\langle 0, k\rangle$. Let $z_{s}=\phi_{\alpha(s)} \cdot h_{s}$ for $s=1, \ldots, m$ with $\alpha$ and $h_{s}$ as above and let

$$
\mathbf{z}_{s}=\left[g\langle 1, k\rangle \cdot z_{s}, \ldots, g\langle k, k\rangle \cdot z_{s}, z_{s}\right] \in E_{k}(G, x) / C_{G}(x) .
$$

The fiber of the cyclic covering $p_{\bullet}^{x}$ over $\Phi_{k}^{x}\left(g_{0}, \ldots, g_{k}\right)$ may then be written in the form $\left\{\mathbf{z}_{1}, \ldots, \mathbf{z}_{m}\right\}$. Using this notation, the value of $\operatorname{Trf}_{k} \circ \Phi_{k}^{x}$ applied to $\left(g_{0}, \ldots, g_{k}\right)$ is represented by

$$
\left(\left(\alpha_{0}, \ldots, \alpha_{k}\right),\left(\mathbf{z}_{1}, \ldots, \mathbf{z}_{m}\right)\right) \in \Sigma_{m}^{k+1} \times\left(E_{k}(G, x) / C_{H}(x)\right)^{m}
$$

with $\alpha_{i}$ as above. We claim that applying the retraction $r_{\bullet}$ to each of the $k$-simplices $\mathbf{z}_{s}$ we exactly get the representative (6.12). In order to see this we write $\mathbf{z}_{s}$ in the form

$$
\begin{aligned}
& \mathbf{z}_{s}=\left[g\langle 1, k\rangle \cdot \phi_{\alpha(s)} \cdot h_{s}, \ldots, g\langle k, k\rangle \cdot \phi_{\alpha(s)} \cdot h_{s}, \phi_{\alpha(s)} \cdot h_{s}\right] \\
& =\left[\phi_{t_{0}} \cdot \overline{g\langle 1, k\rangle}(\alpha(s)) \cdot h_{s}, \ldots, \phi_{t_{k-1}} \cdot \overline{g\langle k, k\rangle}(\alpha(s)) \cdot h_{s}, \phi_{t_{k}} \cdot h_{s}\right] .
\end{aligned}
$$

with $t_{j}=i(g\langle j+1, k\rangle)(\alpha(s))$ for $0 \leq j<k$ and $t_{k}=\alpha(s)$. From this description it easily follows that $r_{k}\left(\mathbf{z}_{s}\right)=\mathbf{y}_{s}$ as claimed.

Proof of Theorem 1.2. The chains of equivalences of cyclotomic spectra represented by the horizontal arrows are obtained by combining the equivalences in Section 4 with a product splitting similar to that in Proposition 6.2. The commutativity of the diagram then follows from (i) in Theorem 6.10. 
Proof of Theorem 1.4. We first use Theorem 6.10 to identify $\operatorname{Res}_{G}^{H}(\lambda, \omega)$ with the realization of the combinatorial transfer up to $C_{r}$-equivariant homotopy. The result then follows from Theorem 5.2.

Given an unbased $C_{r}$-space $X$, we let

$$
\mathrm{H}_{*}^{C_{r}}(X, \mathrm{TH}(R))=\pi_{*}\left(\Sigma_{C_{r}}^{\infty}\left(X_{+}\right) \wedge \mathcal{V} \mathrm{TH}(R)\right)^{C_{r}} .
$$

Proof of Theorem 1.1. By definition, the homology transfer associated to a $C_{r}$-equivariant covering projection $X \rightarrow A$ is induced by the $C_{r}$-equivariant stable map

$$
\operatorname{Trf}_{C_{r}} \wedge \mathrm{id}: \Sigma_{C_{r}}^{\infty}\left(A_{+}\right) \wedge_{\mathcal{V}} \mathrm{TH}(R) \rightarrow \Sigma_{C_{r}}^{\infty}\left(X_{+}\right) \wedge_{\mathcal{V}} \mathrm{TH}(R)
$$

with $\operatorname{Trf}_{C_{r}}$ as in Section 5.2. The result is thus an immediate consequence of Theorem 1.2 and Theorem 1.4.

\section{LAURENT POLYNOMIALS AND TC-TRANSFER INVARIANT ELEMENTS}

The proof of Theorem 1.6 is based on the existence of a transfer compatible TC-assembly map. In its crudest form, the TC-assembly map associated to a discrete group $G$ and a symmetric ring spectrum $R$ is a map in the stable homotopy category of the form

$$
B G_{+} \wedge \mathrm{TC}(R) \rightarrow \mathrm{TC}(R[G]) .
$$

We shall need a version of this which in a strict sense is compatible with transfer maps associated to subgroups of finite index.

7.1. The transfer compatible TC-assembly map. Let $\mathcal{F}[G]$ be the skeleton category of finitely generated free $G$-sets. This is a category with coproducts and we recall in Appendix A.4 how it gives rise to a $\Gamma$-space $\mathcal{D} \mathcal{F}[G]$ whose associated spectrum $\mathrm{K}(\mathcal{F}[G])$ is the algebraic K-theory spectrum of $\mathcal{F}[G]$. By definition, the zeroth space of this spectrum is given by

$$
\coprod_{n \geq 0} B\left(\Sigma_{n} \int G\right)
$$

where $\Sigma_{n} \int G$ denotes the wreath product of $\Sigma_{n}$ acting on $G^{n}$ by permuting the coordinates. The summand corresponding to $n=1$ may be identified with $B G$ and it is a consequence of the Barratt-Priddy-Quillen-Segal Theorem that the associated map of spectra

$$
\Sigma^{\infty}\left(B G_{+}\right) \rightarrow \mathrm{K}(\mathcal{F}[G])
$$

is an equivalence. The advantage of the model $K(\mathcal{F}[G])$ from our point of view is that a subgroup of finite index $H \subseteq G$ gives rise to a restriction functor $\mathcal{F}[G] \rightarrow \mathcal{F}[H]$ and thus a map of spectra

$$
\operatorname{Res}_{G}^{H}: \mathrm{K}(\mathcal{F}[G]) \rightarrow \mathrm{K}(\mathcal{F}[H]) .
$$

Since we work with skeleton categories this requires the choice of a transversal of $H$ in $G$ as in Section 6. It is well-known that this realizes the stable 
transfer of the "covering up to homotopy" $B H \rightarrow B G$. This fact also follows from our general theory, see Remark 7.2. Consider now the composition

$$
\mathrm{K}(\mathcal{F}[G]) \rightarrow \mathrm{K}(\mathbb{S}[G]) \stackrel{\operatorname{trc}}{\rightarrow} \mathrm{TC}(\mathbb{S}[G]),
$$

where the first map is defined in the natural way, see Appendix A.4, and trc is the cyclotomic trace whose definition we recall in Appendix A.3. By definition, trc represents a chain of maps in which each wrong way map is an equivalence. Applying a construction similar to that in Section 2.3 we form the smash product with $\mathrm{TC}(R)$ and using the pairing $\mathbb{S}[G] \wedge R \rightarrow R[G]$, we define the TC-assembly map to be the composition

$$
\alpha: \mathrm{K}(\mathcal{F}[G]) \wedge \mathrm{TC}(R) \rightarrow \mathrm{TC}(\mathbb{S}[G]) \wedge \mathrm{TC}(R) \rightarrow \mathrm{TC}(R[G]) .
$$

It follows from Appendix A.4 that this is natural with respect to transfers in the sense that a subgroup $H \subseteq G$ of finite index gives rise to a commutative diagram of the form

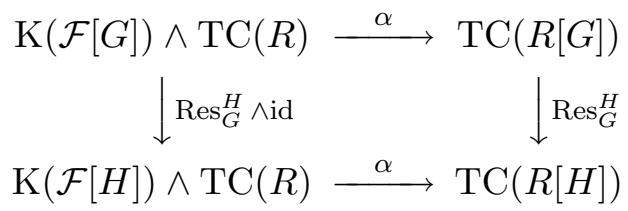

in which the horizontal arrows represent chains of maps each of which commutes strictly with the induced restriction maps.

Proposition 7.1. Suppose that $G$ is a torsion free discrete group and that $B G$ has the homotopy type of a finite $C W$-complex. Then the $T C$-assembly map is split injective in the stable homotopy category.

Proof. Recall the decomposition of the cyclic bar construction in (6.1) and observe that $G$ being torsion free implies that the unit component $B^{\text {cy }}\left(G, 1_{G}\right)$ is a cyclotomic subspace. Using the equivalences in Section 4 we get a chain of maps of cyclotomic spectra

$$
\begin{aligned}
\operatorname{TH}(R[G]) & \simeq \mathbb{E} \Sigma_{\mathbb{T}}^{\infty}\left(B^{\mathrm{cy}}(G)_{+}\right) \wedge_{\mathcal{V}} \mathrm{TH}(R) \\
& \rightarrow \mathbb{E} \Sigma_{\mathbb{T}}^{\infty}\left(B^{\mathrm{cy}}\left(G, 1_{G}\right)_{+}\right) \wedge_{\mathcal{V}} \mathrm{TH}(R) .
\end{aligned}
$$

Restricting a cyclotomic spectrum to the trivial representations $\mathbb{R}^{n}$ gives a spectrum with cyclotomic $\mathbb{T}$-action in the sense of Appendix A.1. The assembly map as defined above is induced by a chain of maps of spectra with cyclotomic $\mathbb{T}$-action of the form

$$
\mathrm{K}(\mathcal{F}[G]) \wedge \mathrm{TH}(R) \rightarrow \mathrm{TH}(R[G])
$$

by passing to the homotopy limit over the $C_{r}$ fixed-point spectra and composing with the canonical map

$$
\mathrm{K}(\mathcal{F}[G]) \wedge \mathrm{TC}(R) \rightarrow \operatorname{holim}\left(\mathrm{K}(\mathcal{F}[G]) \wedge \mathrm{TH}(R)^{C_{r}}\right) .
$$

Here we view $\mathrm{K}(\mathcal{F}[G])$ as a spectrum with trivial cyclotomic $\mathbb{T}$-action. Using the equivalence $\Sigma^{\infty}\left(B G_{+}\right) \rightarrow \mathrm{K}(\mathcal{F}[G])$, it follows from the assumption that $B G$ be of the homotopy type of a finite $\mathrm{CW}$ complex that the last map is 
an equivalence. Thus it suffices to show that the composite chain of maps of spectra with cyclotomic $\mathbb{T}$-action

$$
\mathrm{K}(\mathcal{F}[G]) \wedge \mathrm{TH}(R) \rightarrow \mathbb{E} \Sigma_{\mathbb{T}}^{\infty}\left(B^{\mathrm{cy}}\left(G, 1_{G}\right)_{+}\right) \wedge \mathcal{V} \mathrm{TH}(R)
$$

induces an equivalence on $C_{r}$ fixed-point spectra for all $r$. Since $G$ is torsion free it follows from Example 6.6 that the fixed point inclusion

$$
B^{\mathrm{cy}}\left(G, 1_{G}\right)^{C_{r}} \rightarrow B^{\mathrm{cy}}\left(G, 1_{G}\right)
$$

is a $C_{r}$-equivariant homotopy equivalence for all $r$. Consequently, there are canonical stable equivalences

$$
B^{\mathrm{cy}}\left(G, 1_{G}\right)_{+}^{C_{r}} \wedge \mathrm{TH}(R)^{C_{r}} \simeq\left(\mathbb{E} \Sigma_{\mathbb{T}}^{\infty}\left(B^{\mathrm{cy}}\left(G, 1_{G}\right)_{+}\right) \wedge_{\mathcal{V}} \mathrm{TH}(R)\right)^{C_{r}}
$$

and checking from the definitions one verifies that the diagrams

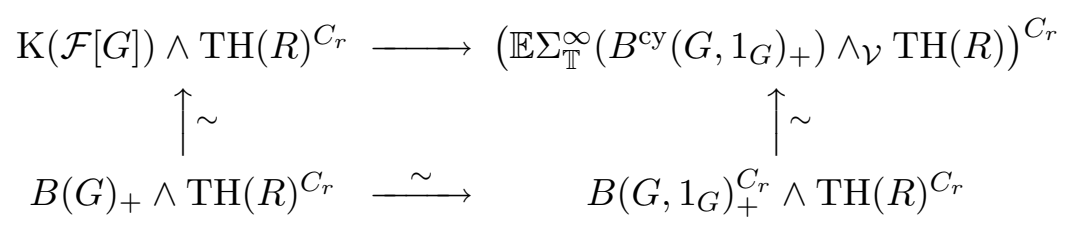

are commutative in the stable homotopy category. This completes the proof.

Remark 7.2. By an argument similar to that in the above proof one shows that the stable map

$$
\mathrm{K}(\mathcal{F}[G]) \rightarrow \mathrm{K}(\mathbb{S}[G]) \rightarrow \mathrm{TH}(\mathbb{S}[G]) \rightarrow \Sigma^{\infty}\left(B^{\mathrm{cy}}\left(G, 1_{G}\right)_{+}\right)
$$

is an equivalence. This splitting is due to Waldhausen [30]. Applying Theorem 1.4 in the special case $r=1, x=1_{H}$ and using that the trace map is natural for restriction maps, we recover the fact that the restriction map $\mathrm{K}(\mathcal{F}[G]) \rightarrow \mathrm{K}(\mathcal{F}[H])$ associated to a subgroup of finite index represents the stable transfer.

7.2. The proof of Theorem 1.6. We first fix $n$ and analyze the action of the TC-transfer $t_{n}$ in (1.5). Let $F\left(t_{n}\right.$, id) be the homotopy equalizer of $t_{n}$ and the identity. As usual for such homotopy limits of $\Omega$-spectra this is defined by evaluating the space level homotopy equalizer in each spectrum degree. In the stable homotopy category, $F\left(t_{n}\right.$, id $)$ is equivalent to the fiber of $t_{n}$ - id and on the level of homotopy groups there results a long exact sequence

$$
\cdots \rightarrow \pi_{i} F\left(t_{n}, \mathrm{id}\right) \rightarrow \pi_{i} \mathrm{TC}\left(R\left[z, z^{-1}\right]\right) \stackrel{t_{n}-\mathrm{id}}{\longrightarrow} \pi_{i} \mathrm{TC}\left(R\left[z, z^{-1}\right]\right) \rightarrow \ldots
$$

It is clear that the subgroup of $t_{n}$-fixed homotopy classes equals the image of $\pi_{*} F\left(t_{n}, \mathrm{id}\right)$. Let $\operatorname{Trf}_{n}$ be the stable transfer

$$
\operatorname{Trf}_{n}: S_{+}^{1} \wedge \mathrm{TC}(R) \rightarrow S_{+}^{1} \wedge \mathrm{TC}(R)
$$


associated to the $n$-fold covering $S^{1} \rightarrow S^{1}$. It follows from Remark 7.2 that this is related to $t_{n}$ by the following commutative diagram of fibre sequences in the stable homotopy category

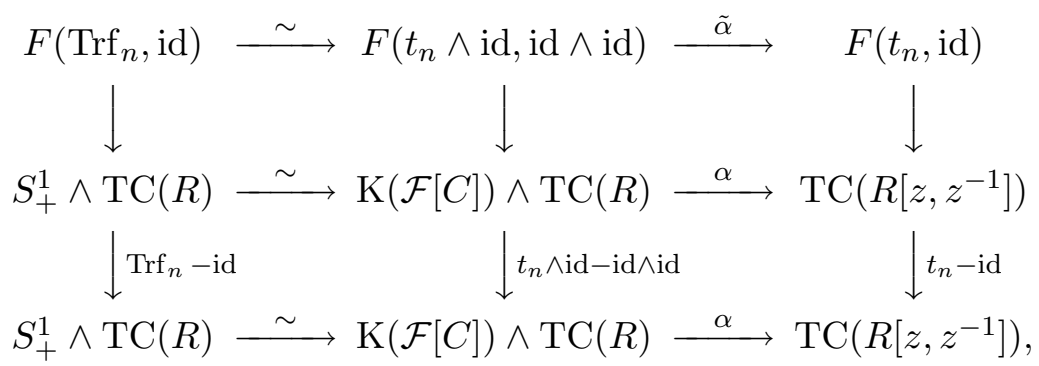

where $\alpha$ is the assembly map associated to the infinite cyclic group $C$. The latter satisfies the conditions in Proposition 7.1 implying that $\alpha$ is split injective.

Lemma 7.3. The stable map of homotopy equalizers $\tilde{\alpha}$ is an equivalence.

Proof. As in the proof of Proposition 7.1 we shall work in the setting of spectra with cyclotomic $\mathbb{T}$-action as opposed to cyclotomic spectra. In the proof we let $t_{n}$ be the restriction map on $\operatorname{TH}\left(R\left[z, z^{-1}\right]\right)$ and write $t_{n}^{\mathrm{TC}}$ for the induced map on $\mathrm{TC}\left(R\left[z, z^{-1}\right]\right)$. Thus $t_{n}^{\mathrm{TC}}$ is now what was denoted $t_{n}$ above and $F\left(t_{n}\right.$, id $)$ now denotes the homotopy equalizer of $t_{n}$ and the identity on $\operatorname{TH}\left(R\left[z, z^{-1}\right]\right)$. Notice that $F\left(t_{n}\right.$, id $)$ inherits a cyclotomic $\mathbb{T}$-action from that of $\operatorname{TH}\left(R\left[z, z^{-1}\right]\right)$ and that the fixed-point spectrum $F\left(t_{n}, \mathrm{id}\right)^{C_{r}}$ may be identified with $F\left(t_{n}^{C_{r}}, \mathrm{id}^{C_{r}}\right)$. Since homotopy limits commute we have that

$$
F\left(t_{n}^{\mathrm{TC}}, \mathrm{id}^{\mathrm{TC}}\right)=\underset{\mathbb{I}}{\operatorname{holim}} F\left(t_{n}^{C_{r}}, \mathrm{id}^{C_{r}}\right) .
$$

It is because of these functorial properties that we introduce homotopy equalizers in the proof. In a similar way, the homotopy equalizer of the diagram

$$
\mathrm{K}(\mathcal{F}[C]) \wedge \mathrm{TH}(R) \stackrel{t_{n} \wedge \mathrm{id}, \mathrm{id} \wedge \mathrm{id}}{\rightrightarrows} \mathrm{K}(\mathcal{F}[C]) \wedge \mathrm{TH}(R)
$$

inherits a cyclotomic $\mathbb{T}$-action from from that of $\mathrm{TH}(R)$. By an argument similar to that in the proof of Proposition 7.1, it suffices to show that this is related to $F\left(t_{n}\right.$, id $)$ by a chain of equivalences. Letting $z$ be the preferred generator of the infinite cyclic group $C$, the cyclic bar construction $B^{\text {cy }}(C)$ splits as a disjoint union of the subspaces $B^{\mathrm{cy}}\left(C, z^{d}\right)$, where $d$ takes all integer values. Applying Theorem 1.2 we get an equivalence of spectra with cyclotomic $\mathbb{T}$-action

$$
\mathrm{TH}\left(R\left[z, z^{-1}\right]\right) \simeq \prod_{d=-\infty}^{\infty} \mathbb{E} \Sigma_{\mathbb{T}}^{\infty}\left(B^{\mathrm{cy}}\left(C, z^{d}\right)_{+}\right) \wedge_{\mathcal{V}} \mathrm{TH}(R),
$$

where $t_{n}$ maps the factors indexed by integers $d$ not divisible by $n$ to the base point and the factor indexed by $n d$ to the factor indexed by $d$. It follows that the stable map $t_{n}-$ id restricts to an equivalence on the weak 
product of the factors indexed by $d \neq 0$. This holds for the induced stable map $t_{n}^{C_{r}}-\mathrm{id}^{C_{r}}$ on fixed-point spectra as well. As in the proof of Proposition 7.1 we consider the chain of maps

$$
\mathrm{TH}\left(R\left[z, z^{-1}\right]\right) \rightarrow \mathbb{E} \Sigma_{\mathbb{T}}^{\infty}\left(B^{\mathrm{cy}}\left(C, 1_{C}\right)_{+}\right) \wedge_{\mathcal{V}} \mathrm{TH}(R),
$$

induced by the projection onto the cyclotomic subspace $B^{\mathrm{cy}}\left(C, 1_{C}\right)$. It follows from the above remarks that $F\left(t_{n}\right.$, id) is equivalent to the homotopy equalizer of $t_{n} \wedge$ id and id $\wedge$ id acting on the right hand side. Furthermore, by an argument similar to that used in the proof of Proposition 7.1, we may use the assembly map to identify the right hand side with $\mathrm{K}(\mathcal{F}[C]) \wedge \mathrm{TH}(R)$ as a spectrum with cyclotomic $\mathbb{T}$-action. Since the assembly map is compatible with transfers this concludes the proof.

Corollary 7.4. The assembly map induces an isomorphism of the transfer invariant subgroups

$$
\left\{\pi_{*} S_{+}^{1} \wedge \mathrm{TC}(R)\right\}^{\operatorname{Trf}_{n}} \stackrel{\sim}{\rightarrow}\left\{\pi_{*} \operatorname{TC}\left(R\left[z, z^{-1}\right]\right)\right\}^{t_{n}}
$$

for each $n$.

Proof of Theorem 1.6. Consider the stable map

$$
\Sigma^{\infty}\left(S^{1}\right) \stackrel{\{\eta, \text { id }\}}{\longrightarrow} \Sigma^{\infty}\left(S^{0}\right) \vee \Sigma^{\infty}\left(S^{1}\right) \simeq \Sigma^{\infty}\left(S_{+}^{1}\right),
$$

where $\eta$ denotes the stable Hopf map. Smashing with $\mathrm{TC}(R)$ and composing with the assembly map we get a stable map

$$
S^{1} \wedge \mathrm{TC}(R) \rightarrow S_{+}^{1} \wedge \mathrm{TC}(R) \stackrel{\alpha}{\rightarrow} \mathrm{TC}\left(R\left[z, z^{-1}\right]\right) .
$$

Here we use the chosen generator $z$ of $C$ to identify $B C$ with $S^{1}$. The induced map of homotopy groups

$$
\pi_{i-1} \mathrm{TC}(R) \simeq \pi_{i} S^{1} \wedge \mathrm{TC}(R) \rightarrow \pi_{i} \mathrm{TC}\left(R\left[z, z^{-1}\right]\right)
$$

is then multiplication by $u$ as in the statement of the theorem. Using the stable splitting

$$
S_{+}^{1} \wedge \mathrm{TC}(R) \simeq \mathrm{TC}(R) \vee S^{1} \wedge \mathrm{TC}(R),
$$

it is well-known that the transfer $\operatorname{Trf}_{n}$ is represented by the $2 \times 2$-matrix

$$
\left(\begin{array}{cc}
n & (n-1) \eta \\
0 & 1
\end{array}\right)
$$

On the level of homotopy groups we see that the image of the first map in (7.5) is contained in the $\operatorname{Trf}_{n}$ fixed-set and the image of the composite map is thus contained in the $t_{n}$ fixed-set. This holds for all $n$, so the image is in fact contained in the transfer invariant subgroup. On the other hand, using Corollary 7.4 and letting $n=2$ we see that the image is exactly the subgroup of invariants.

Remark 7.6. It follows from the proof that the transfer invariant subgroup equals the set of homotopy classes fixed by $t_{2}$. 


\section{Appendix A. Restriction maps AND the CyClotomiC trace}

In this appendix we verify the claim made in the introduction that the cyclotomic trace trc is natural with respect to restriction maps. We also provide the necessary background for the construction of the assembly map used in Section 7.1. The main problem is to define trc in a way that is sufficiently natural with respect to functors on the categories $\mathcal{F}(R)$ of finitely generated free $R$-modules. Here we shall follow the exposition in [28], which gives a streamlined version of the original construction in [8]. The construction is based on Goodwillie's notion of a cyclotomic spectrum which we recall first.

A.1. Epicyclic and cyclotomic spectra. The definition of an epicyclic space is based on the edgewise subdivision functors $\operatorname{sd}_{r}$ from [8, Section 1]. Recall that $\operatorname{sd}_{r}$ takes a cyclic space $Z_{\bullet}$ to the simplicial $C_{r}$-space $\operatorname{sd}_{r} Z \bullet$ and that the simplicial fixed-point space $\operatorname{sd}_{r} Z_{\bullet}^{C_{r}}$ inherits a cyclic action from that of $Z_{\bullet}$.

Definition A.1 (Goodwillie [12]). An epicyclic space is a cyclic space $X$. equipped with a family of cyclic maps $R_{r}: \operatorname{sd}_{r} X_{\bullet}^{C_{r}} \rightarrow X_{\bullet}$ for $r \geq 1$ such that (i) $R_{1}=$ id and (ii) the diagrams

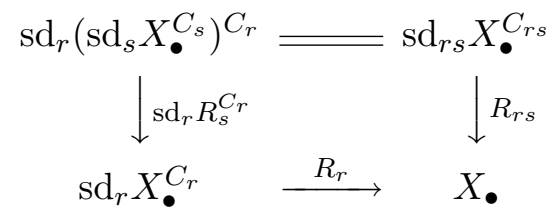

are commutative. Similarly, an epicyclic spectrum is a cyclic spectrum equipped with a family of cyclic maps $R_{r}$ as above satisfying (i) and (ii) in each spectrum degree.

We shall introduce a $\mathbb{T}$-equivariant analogue of this and let $\rho_{r}: \mathbb{T} \rightarrow \mathbb{T} / C_{r}$ be the homeomorphism from Section 2.2.

Definition A.2. A cyclotomic space is a $\mathbb{T}$-space $X$ equipped with a family of $\mathbb{T}$-equivariant maps $R_{r}: \rho_{r}^{*} X^{C_{r}} \rightarrow X$ for $r \geq 1$ such that (i) $R_{1}=$ id and (ii) the diagrams

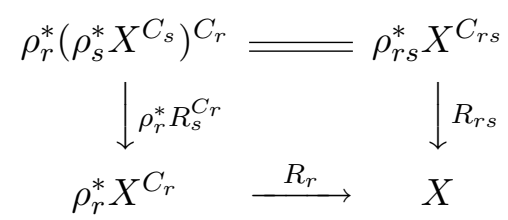

are commutative. In accordance with the notation in Section 2.1, we call the spectrum version of this a spectrum with cyclotomic $\mathbb{T}$-action.

An epicyclic structure on a cyclic space or spectrum $X_{\bullet}$ induces a cyclotomic structure on the topological realization $\left|X_{\bullet}\right|$. This uses that the natural homeomorphisms $D_{r}$ from $[8,1.1]$ restrict to $\mathbb{T}$-equivariant homeomorphisms $\left|\operatorname{sd}_{r} X_{\bullet}^{C_{r}}\right| \rightarrow \rho_{r}^{*}\left|X_{\bullet}\right|^{C_{r}}$ when the domain is given the $\mathbb{T}$-action 
induced by the cyclic structure of $\operatorname{sd}_{r} X_{\bullet}^{C_{r}}$. The cyclotomic structure maps are then defined by

$$
R_{r}: \rho_{r}^{*}\left|X_{\bullet}\right|^{C_{r}} \cong\left|\operatorname{sd}_{r} X_{\bullet}^{C_{r}}\right| \rightarrow\left|X_{\bullet}\right|
$$

in which the last maps are obtained by realizing the epicyclic structure maps of $X_{\bullet}$. Let now $R$ be a symmetric ring spectrum and recall from Section 3.1 that the definition of $\mathrm{TC}(R)$ only uses the structure of $\mathrm{TH}(R)$ as a spectrum with cyclotomic $\mathbb{T}$-action, not the full structure as a cyclotomic $\mathbb{T}$-spectrum. Furthermore, this structure is induced by an epicyclic structure on $\mathrm{TH}_{\bullet}(R)$. In order to define the cyclotomic trace we first consider a chain of maps of epicyclic spectra

$$
\operatorname{tr}_{\bullet}: \mathrm{K}(R) \rightarrow \mathrm{TH}_{\bullet}(R),
$$

where we view the algebraic K-theory spectrum $\mathrm{K}(R)$ as a constant epicyclic spectrum and each wrong way map in the chain is an equivalence. Passing to topological realizations and evaluating the associated homotopy limits over II we get the chain of maps

$$
\operatorname{trc}: \mathrm{K}(R) \rightarrow \mathrm{TC}(R)
$$

representing the cyclotomic trace. We shall recall the definition of $\mathrm{K}(R)$ in Appendix A.2 and in Section A.3 we outline the chain of maps defining tr. and show that each map in the chain is compatible with a suitably defined restriction map. The definition of $\mathrm{K}(R)$ is based on Segal's notion of $\Gamma$-spaces [29], [6]. We recall that a $\Gamma$-space $A$ is a base point preserving functor from finite based sets to based spaces and that there is a functorial way to associate a spectrum $A(\mathbb{S})$ to such a functor.

A.2. Algebraic K-theory of symmetric ring spectra. As in Section 3.1 we let $\mathcal{F}$ be the skeleton category of finite based sets of the form $\vee_{i=1}^{r} S^{0}$. This is a category with coproducts, thus give rise to the $\Gamma$-category $\mathcal{D} \mathcal{F}$ that to a finite based set $X$ associates the category $\mathcal{D} \mathcal{F}(X)$ of sum diagrams $\theta=\left\{\theta_{U}\right\}$ in $\mathcal{F}$ indexed on subsets $U$ of $\bar{X}=X-\{*\}$, see eg [28]. Given such a sum diagram $\theta$, we write $\theta_{x}$ for the object in $\mathcal{F}$ indexed by the singleton $\{x\} \subseteq \bar{X}$. Let now $R$ be a symmetric ring spectrum and let $\mathcal{F}(R)$ be the skeleton category of finitely generated free $R$-modules introduced in Section 3.1. As usual we write the objects in the form $R(S)=R \wedge S$, where $S$ is an object in $\mathcal{F}$. Recall that this is a category enriched in symmetric spectra in the sense that there is a symmetric spectrum of morphisms $M\left(R(S), R\left(S^{\prime}\right)\right)$ relating $R(S)$ and $R\left(S^{\prime}\right)$. We extend this to a $\Gamma$-category $\mathcal{D} \mathcal{F}(R)$ enriched in symmetric spectra in which the objects of $\mathcal{D} \mathcal{F}(R)(X)$ have the form $R(\theta)=R \wedge \theta$, for $\theta$ an object of $\mathcal{D} \mathcal{F}(X)$. Given objects $R(\theta)$ and $R\left(\theta^{\prime}\right)$, we define $M\left(R(\theta), R\left(\theta^{\prime}\right)\right)$ to be the symmetric spectrum

$$
\prod_{x \in \bar{X}} M\left(R\left(\theta_{x}\right), R\left(\theta_{x}^{\prime}\right)\right)
$$


The $\Gamma$-structure is inherited from that of $\mathcal{D F}(X)$, see [28]. Notice that the above spectrum only depends on the values $\theta_{x}$ and $\theta_{x}^{\prime}$ and that if $\theta_{x}=\theta_{x}^{\prime}$ for all $x \in \bar{X}$, then it is a product of symmetric ring spectra, hence itself a symmetric ring spectrum. From this $\Gamma$-category we construct a topological $\Gamma$-category $G L(\mathcal{D F}(R))$ with the same objects. The morphism space

$$
G L\left(R(\theta), R\left(\theta^{\prime}\right)\right) \subseteq Q_{\mathcal{I}}\left(M\left(R(\theta), R\left(\theta^{\prime}\right)\right)\right.
$$

is defined to be the group-like submonoid of homotopy invertible components if $\theta_{x}=\theta_{x}^{\prime}$ for all $x \in \bar{X}$ and is the empty set otherwise. Applying the classifying space construction, we get a $\Gamma$-space whose associated spectrum is the algebraic K-theory spectrum of $\mathcal{F}(R)$,

$$
\mathrm{K}(R)=B G L(\mathcal{D F} \mathcal{F}(R))(\mathbb{S}) .
$$

Applying the cyclic classifying space construction, we get a $\Gamma$-space whose associated spectrum is the cyclic algebraic K-theory of $\mathcal{F}(R)$,

$$
\mathrm{K}^{\mathrm{cy}}(R)=B^{\mathrm{cy}} G L(\mathcal{D F}(R))(\mathbb{S}) .
$$

By definition, this is the topological realization of the epicyclic spectrum $\mathrm{K}_{\bullet}^{\mathrm{cy}}(R)$. Notice that each of the topological categories $G L(\mathcal{D F}(R))(X)$ is group-like in the sense that the morphism spaces are either empty or grouplike monoids. For each such group-like category $\mathcal{C}$, the (constant epicyclic) space $B \mathcal{C}$ is related to the epicyclic space $B_{\bullet}^{\text {cy }} \mathcal{C}$ via a natural chain of epicyclic maps $B \mathcal{C} \rightarrow B_{\bullet}^{\text {cy }} \mathcal{C}$ in which each wrong way map is an equivalence, see [12], [28]. Applying this in the case at hand, we get a natural chain of maps of epicyclic spectra $\mathrm{K}(R) \rightarrow \mathrm{K}_{\bullet}^{\mathrm{cy}}(R)$, where we view $\mathrm{K}(R)$ as the constant epicyclic spectrum.

A.3. Restriction maps and the cyclotomic trace. In order to relate K-theory to topological Hochschild homology we now extend the definition of $\mathrm{TH}_{\bullet}(R)$ to a $\Gamma$-object in the category of epicyclic spectra by applying the construction in Section 3.1 to each of the categories $\mathcal{D} \mathcal{F}(R)(X)$, that is,

$$
\mathrm{TH}_{\bullet}(\mathcal{D} \mathcal{F}(R))(X)=\mathrm{TH}_{\bullet}(\mathcal{D F}(R)(X)) \text {. }
$$

This gives rise to an epicyclic bispectrum TH. $(\mathcal{D F}(R))(\mathbb{S})$ with one spectrum direction coming from the $\Gamma$-structure and the other from the THconstruction. Setting the spectrum coordinate arising from the $\Gamma$-structure equal to zero we recover the spectrum $\mathrm{TH}(R)$. Let us write $\mathrm{TH}_{\bullet}^{\prime}(R)$ for the spectrum obtained by setting the TH-spectrum coordinate equal to zero. Since TH. $(\mathcal{D F}(R))(\mathbb{S})$ is an $\Omega$-bispectrum in each simplicial degree, it follows by a standard argument that these two spectra are related by a chain of equivalences of epicyclic spectra

$$
\mathrm{TH}_{\bullet}^{\prime}(R) \simeq \mathrm{TH}_{\bullet}(R) .
$$


By definition, $\mathrm{TH}_{\bullet}^{\prime}(R)$ is the spectrum associated to the $\Gamma$-epicyclic space that to a finite based set $X$ associates the epicyclic space

$[k] \mapsto \underset{\mathcal{I}^{k+1}}{\operatorname{hocolim}} \Omega^{n_{0}+\cdots+n_{k}}\left(\bigvee_{\theta_{0}, \ldots, \theta_{k}} M\left(R\left(\theta_{0}\right), R\left(\theta_{k}\right)\right) \wedge \cdots \wedge M\left(R\left(\theta_{k}\right), R\left(\theta_{k-1}\right)\right)\right)$,

where $\theta_{0}, \ldots, \theta_{k}$ runs through all $(k+1)$-tuples of objects in $\mathcal{D} \mathcal{F}(X)$. Forming smash product of maps in the usual way we get a natural map of epicyclic spectra $\mathrm{K}_{\bullet}^{\mathrm{cy}}(R) \rightarrow \mathrm{TH}_{\bullet}^{\prime}(R)$, induced by a map of the underlying $\Gamma$-epicyclic spaces. The chain of maps represented by tr. in (A.3) is then defined to be the composition

$$
\operatorname{tr}_{\bullet}: \mathrm{K}(R) \rightarrow \mathrm{K}_{\bullet}^{\mathrm{cy}}(R) \rightarrow \mathrm{TH}_{\bullet}^{\prime}(R) \simeq \mathrm{TH}_{\bullet}(R) .
$$

Suppose now that $f: \Pi \rightarrow \Upsilon$ is a map of based monoids and $\phi: \Pi^{\vee n} \rightarrow \Upsilon$ a specified isomorphism of right $\Pi$-sets as in Section 4.1. Then the restriction functor $f^{*}$ defined in that section has a canonical extension to a functor of $\Gamma$-categories enriched in symmetric spectra

$$
f^{*}: \mathcal{D} \mathcal{F}(R(\Upsilon)) \rightarrow \mathcal{D} \mathcal{F}(R(\Pi))
$$

inducing the restriction map in algebraic K-theory,

$$
f^{*}: \mathrm{K}(R(\Upsilon)) \rightarrow \mathrm{K}(R(\Pi))
$$

Proposition A.4. There is a diagram of epicyclic spectra

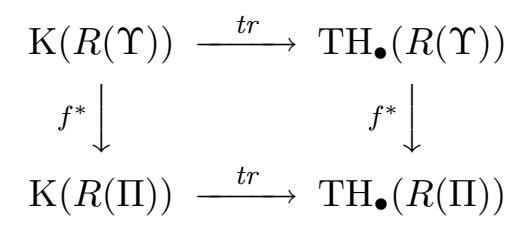

in which tr represents a chain of maps of epicyclic spectra each of which commute strictly with the restriction maps $f^{*}$.

Proof. Consider the diagram

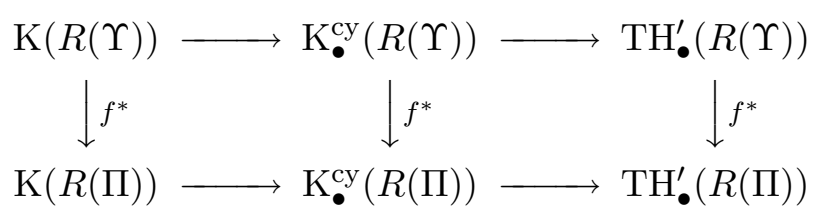

in which the vertical maps are induced by the functor of $\Gamma$-categories $f^{*}$ introduced above. The horizontal arrows represents chains of maps each of which are natural with respect to such functors. The same holds for the equivalences relating $\mathrm{TH}^{\prime}$ and $\mathrm{TH}$ and the conclusion follows.

Passing to topological realization and homotopy limits, it follows that the cyclotomic trace commutes with $f^{*}$ as claimed. 
A.4. Free monoid actions on sets and restriction maps. In this section we provide the background needed for the definition of the TC-assembly map in Section 7.1. Given a based monoid $\Pi$, let $\mathcal{F}(\Pi)$ be the skeleton category of finitely generated free right $\Pi$-sets of the form $\Pi \wedge S$, where $S$ is an object in $\mathcal{F}$. We extend this to a $\Gamma$-category $\mathcal{D} \mathcal{F}(\Pi)$ in which the objects of $\mathcal{D} \mathcal{F}(\Pi)(X)$ are $\mathcal{P}(\bar{X})$-diagrams in $\mathcal{F}(\Pi)$ of the form $\Pi \wedge \theta$, where $\theta$ is an object in $\mathcal{D} \mathcal{F}(X)$. A morphism is a natural isomorphism of such diagrams in $\mathcal{F}(\Pi)$. Applying the classifying space construction we get a $\Gamma$-space $B \mathcal{D} \mathcal{F}(\Pi)$ whose associated spectrum

$$
\mathrm{K}(\mathcal{F}(\Pi))=B \mathcal{D} \mathcal{F}(\Pi)(\mathbb{S})
$$

is the algebraic K-theory spectrum of $\mathcal{F}(\Pi)$. Notice that this spectrum is equivalent to the one associated to the $\Gamma$-space obtained by allowing all coproducts in $\mathcal{F}(\Pi)$. It follows from the Barratt-Priddy-Quillen-Segal theorem that $\mathrm{K}(\mathcal{F}(\Pi))$ is equivalent to the suspension spectrum $\Sigma^{\infty}\left(B \Pi_{+}^{*}\right)$, where $\Pi^{*}$ denotes the units of $\Pi$. Let now $f: \Pi \rightarrow \Upsilon$ and $\phi$ be as in Section 4.1. As in the case of monoid ring spectra, these data determine a restriction functor of $\Gamma$-categories inducing a restriction map in algebraic K-theory. It follows from the definitions that this restriction map is related to that for monoid ring spectra by a commutative diagram

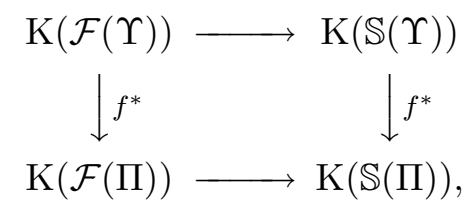

where the horizontal maps are induced by the natural inclusions of the underlying $\Gamma$-categories. When $G$ is a discrete group we write $\mathcal{F}[G]=\mathcal{F}\left(G_{+}\right)$ and $\operatorname{Res}_{G}^{H}$ for the restriction map corresponding to a subgroup of finite index. The claim in Section 7.1 that the TC-assembly map commutes with restriction maps now follows from the above diagram together with Proposition A.4

\section{REFERENCES}

[1] J.F. Adams, Stable homotopy and generalized homology, Chicago Lectures in Mathematics, University of Chicago University Press, 1974.

[2] J.F. Adams, Infinite loop spaces, Annals of Mathematics Studies 90, Princeton University Press, 1978.

[3] H. Bass, Algebraic K-theory, W. A. Benjamin, New York, 1968.

[4] M. Barratt and P. Eccles, $\Gamma^{+}$-structures-I: A free group functor for stable homotopy theory, Topology 13, (1974), pp. 25-45.

[5] S. Betley and C. Schlichtkrull, The cyclotomic trace and curves on K-theory, to appear in Topology.

[6] A.K. Bousfield and E.M. Friedlander, Homotopy theory of $\Gamma$-spaces, spectra, and bisimplicial sets, from: "Geometric applications of homotopy theory (Proc. Conf. Evanstone, Ill, 1977) II", (M.G. Barratt, M.E. Mahowald editors) Springer LNM 658, Springer, Berlin (1978), pp. 80-130.

[7] M. Bökstedt, Topological Hochschild homology, Preprint (1985), Bielefeld. 
[8] M. Bökstedt, W.C. Hsiang and I. Madsen, The cyclotomic trace and algebraic Ktheory of spaces, Invent. Math. 111 (1993), pp. 865-940.

[9] B. Dundas, Relative K-theory and topological cyclic homology, Acta. Math. 179 no. 2, (1997), pp. 223-242.

[10] B. Dundas, The cyclotomic trace for symmetric monoidal categories, from: "Geometry and topology: Aarhus (1998)", Contemp. Math., 258, Amer. Math. Soc. 2000, pp. 121-143.

[11] B. Dundas and R. McCarthy, Topological Hochschild homology of ring functors and exact categories. J. Pure Appl. Algebra 109 (1996), pp. 231-294.

[12] T. Goodwillie, Notes on the cyclotomic trace, Lecture notes, 1990.

[13] H. Hauschild, Zerspaltung äquivarianter Homotopiemengen, Math. Ann. 230, (1977), pp. 279-292.

[14] L. Hesselholt and I. Madsen, On the K-theory of finite algebras over Witt vectors of finite fields, Topology 36, No. 1, (1997), pp. 29-101.

[15] M. Hovey, B. Shipley and J. Smith, Symmetric spectra, J. Amer. Math. Soc. 13 (2000), pp. 149-208.

[16] L.G. Lewis, J.P. May and M. Steinberger, Equivariant stable homotopy theory, Springer LNM 1213, Springer Verlag, 1986.

[17] M. Lydakis, Free loop spaces and equivariant classifying spaces Arch. Math. 77, no. 2, (2001), pp. 181-186.

[18] S. Mac Lane, Categories for the working mathematician, Graduate Texts in Mathematics 5, Springer Verlag, New York-Berlin, 1971.

[19] R. McCarthy, The cyclic homology of an exact category, J. Pure Appl. Algebra 93, (1994), no. 3, pp. 251-296.

[20] R. McCarthy, Relative algebraic K-theory and topological cyclic homology, Acta. Math. 179 no. 2 (1997), pp. 197-222.

[21] I. Madsen, Algebraic K-theory and traces, from: "Current Developments in Mathematics, 1995, (Cambridge, MA)", International Press, Cambridge, MA, 1996, pp. 191-321.

[22] M.A. Mandell, J.P. May, S. Schwede and B. Shipley, Model categories of diagram spectra, Proc. London Math. Soc. (3), 82, no. 2, (2001), pp. 441-512.

[23] J.P. May, The geometry of iterated loop spaces, Springer LNM 271, Springer Verlag, 1972.

[24] J.P. May, $E_{\infty}$-spaces, group completion and permutative categories, from "New developments in Topology (Proc. Sympos. Algebraic Topology, Oxford, 1972)", London Math. Soc. Lecture Notes 11, Cambridge University Press, pp. 61-93.

[25] A. Ranicki, Lower K- and L-Theory, London Math. Soc. Lecture Notes 178, Cambridge University Press, 1992.

[26] C. Schlichtkrull, The transfer map in topological Hochschild homology, J. Pure Appl. Algebra 133 (1998), no. 3, pp. 289-316.

[27] C. Schlichtkrull, A discrete model of equivariant stable homotopy theory for cyclic groups, Math. Scand. 85 (1999), pp. 5-29.

[28] C. Schlichtkrull, Cyclic K-theory and the cyclotomic trace, In preparation, see http://www . math.uio.no/ ${ }^{k r u l l / . ~}$

[29] G. Segal, Categories and cohomology theories, Topology 13 (1974), pp. 293-312.

[30] F. Waldhausen, Algebraic K-theory of topological spaces II, Springer LNM 763, Springer Verlag, 1979, pp. 356-394.

[31] F. Waldhausen, Operations in the algebraic K-theory of spaces, Springer LNM 967, Springer Verlag 1982, pp. 390-409.

Department of Mathematics, University of Oslo, PO Box 1053, Blindern, NO-0316 OSLO, NORWAY

E-mail address: krull@math.uio.no 